\title{
SiO collimated outflows driven by high-mass YSOs in G24.78+0.08
}

\author{
C. Codella ${ }^{1,2}$, M. T. Beltrán ${ }^{1}$, R. Cesaroni ${ }^{1}$, L. Moscadelli $^{1}$, R. Neri ${ }^{3}$, M. Vasta ${ }^{1}$, and Q. Zhang ${ }^{4}$
}

\author{
1 INAF, Osservatorio Astrofisico di Arcetri, Largo E. Fermi 5, 50125 Firenze, Italy \\ e-mail: codella@arcetri.astro.it \\ 2 UJF - Grenoble 1/CNRS-INSU, Institut de Planétologie et d'Astrophysique de Grenoble (IPAG) UMR 5274, 38041 Grenoble, \\ France \\ 3 IRAM, 300 rue de la Piscine, 38406 Saint Martin d'Hères, France \\ ${ }^{4}$ Harward-Smithsonian Center for Astrophysics, 60 Garden Street, MA 02138 Cambridge, USA
}

Received 26 June 2012 / Accepted 20 November 2012

\begin{abstract}
Context. The region G24.78+0.08, which is associated with a cluster of high-mass young stellar objects in different evolutionary stages, is one of the best laboratories to investigate massive star formation.

Aims. We aim to image the molecular outflows towards G24.78+0.08 at high-angular resolution using SiO emission, which is considered the classical tracer of protostellar jets. In this way we study the mass loss process in which we previously detected a hypercompact ionised region, as well as rotation and infall signatures.

Methods. We performed SiO observations with the VLA interferometer in the $J=1-0 v=0$ transition and with the SMA array in the 5-4 transition. A complementary IRAM 30-m single-dish survey in the (2-1), (3-2), (5-4), and (6-5) SiO lines was also carried out. Results. Two collimated $\mathrm{SiO}$ high-velocity (up to $25 \mathrm{~km} \mathrm{~s}^{-1}$ w.r.t. the systemic velocity) outflows driven by the A2 and C millimeter continuum massive cores have been imaged. On the other hand, we detected no SiO outflow driven by the young stellar objects in more evolved evolutionary phases that are associated with ultracompact (B) or hypercompact (A1) HII regions. The A2 outflow has also been traced using $\mathrm{H}_{2} \mathrm{~S}$. The LVG analysis of the $\mathrm{SiO}$ emission reveals high-density gas $\left(10^{3}-10^{4} \mathrm{~cm}^{-3}\right)$, with well constrained $\mathrm{SiO}$ column densities $\left(0.5-1 \times 10^{15} \mathrm{~cm}^{-2}\right)$. The driving source of the $\mathrm{A} 2$ outflow is associated with typical hot core tracers such as $\mathrm{CH}_{3} \mathrm{OCHO}$ (methyl formate), $\mathrm{C}_{2} \mathrm{H}_{3} \mathrm{CN}$ (vinyl cyanide), $\mathrm{HCC}^{13} \mathrm{CN}$ (cyanoacetilene), and $\left(\mathrm{CH}_{3}\right)_{2} \mathrm{CO}$ (acetone).

Conclusions. The driving source of the main $\mathrm{SiO}$ outflow in $\mathrm{G} 24$ has an estimated luminosity of a few $10^{4} L_{\odot}$ (typical of a late O-type star) and is embedded in the $1.3 \mathrm{~mm}$ continuum core $\mathrm{A} 2$, which in turn is located at the centre of a hot core that rotates on a plane perpendicular to the outflow main axis. The present $\mathrm{SiO}$ images support a scenario similar to the low-mass case for massive star formation, where jets that are clearly traced by $\mathrm{SiO}$ emission, create outflows of swept-up ambient gas usually traced by $\mathrm{CO}$.
\end{abstract}

Key words. ISM: individual objects: G24.78+0.08 - ISM: molecules - stars: formation

\section{Introduction}

Two main theoretical scenarios, based on accretion, are proposed to explain the formation of O-B type stars: (i) the core accretion model (McKee \& Tan 2002, 2003), where massive stars form from massive cores; and (ii) the competitive accretion model (Bonnell et al. 2007), where a molecular cloud fragments into low-mass cores, which form stars that compete to accrete mass from a common gas reservoir. Both models predict the existence of accretion disks around massive young stellar objects (YSOs), and the presence of jets driving molecular outflows. The core accretion model is a scaled-up scenario of low-mass star formation. The competitive accretion model suggests that massive stars always form in densely clustered environments and that disks and collimated jets are perturbated by interaction with stellar companions. Observation of YSOs with disk/jet systems, and of their properties, would help to distinguish between models.

The region G24.78+0.08 (hereafter G24), located at $7.7 \mathrm{kpc}$ from the Sun, is one of the best laboratories to investigate the process of massive star formation. Several observational campaigns were performed with single-dish antennas and interferometers (Codella et al. 1997; Furuya et al. 2002; Cesaroni et al. 2003; Beltrán 2004, 2005, 2006, 2007, 2011; Moscadelli et al. 2007; Vig et al. 2008) toward this region. G24 is associated with a cluster of high-mass YSOs in different evolutionary stages, distributed in a region with size $\sim 10^{\prime \prime}$ (see YSOs labelled A1, A2, B, C, and D in Fig. 1). Beltrán et al. (2005, 2011) resolved $\mathrm{A} 1$ and $\mathrm{A} 2$ into five cores (see labels in Fig. 2) aligned in a southeast-northwest direction, which suggests a preferential direction for star formation in this region. The A cores are embedded in huge $(0.1 \mathrm{pc})$, massive (a few $100 M_{\odot}$ ) toroids, that rotate around the southeast-northwest (SE-NW) direction, and could still host elusive accretion disks in their interior (Beltrán et al. 2005). This possibility would be even more intriguing for the object labelled A1, which is unique, because of the simultaneous presence of almost all the "ingredients" expected in a typical star formation "recipe": a $20 M_{\odot}$ star surrounded by a hypercompact HII region, that is located at the centre of a rotating toroid undergoing infall towards the star. The association of the rotation of toroids with the coherent motion of accretion disks would be supported by the detection of outflows, possibly driven by jets, along the SE-NW direction. The occurrence of outflows towards A and C has been first reported by Furuya et al. (2002) and has recently been imaged by Beltrán et al. (2011) at high-angular resolution using CO emission. In particular, Beltrán et al. (2011) suggested that $\mathrm{A} 2$, and not $\mathrm{A} 1$, is the driving source of a bipolar $\mathrm{CO}$ outflow located along the SE-NW direction. However, given the complex outflow structure towards cores A1 and A2, we cannot entirely discard the possibility that the core A1 could be powering an additional outflow in the region. 
Table 1. List of the continuum and $\mathrm{SiO}$ observations.

\begin{tabular}{lccccccccc}
\hline \hline Observation & Telescope & $\begin{array}{c}v^{a} \\
(\mathrm{GHz})\end{array}$ & $\begin{array}{c}E_{\mathrm{u}}{ }^{a} \\
(\mathrm{~K})\end{array}$ & $\begin{array}{c}S \mu^{2 a} \\
\left(\mathrm{D}^{2}\right)\end{array}$ & $\begin{array}{c}\text { HPBW } \\
(\mathrm{arcsec})\end{array}$ & $\begin{array}{c}\text { PA } \\
(\mathrm{deg})\end{array}$ & $\begin{array}{c}\text { Spectral resolution } \\
\left(\mathrm{km} \mathrm{s}^{-1}\right)\end{array}$ & $\begin{array}{c}\text { rms noise }^{b} \\
\left(\mathrm{mJy} \mathrm{beam}^{-1}\right)\end{array}$ \\
\hline continuum & VLA-C+D & 43.339 & - & - & $1.5 \times 1.1$ & -11 & - & 0.8 & 309 \\
continuum & SMA & 219.601 & - & - & $1.5 \times 1.4$ & 74 & - & 6.0 & 72 \\
$\mathrm{SiO}(1-0)$ & VLA-D & 43.424 & 2 & 9.6 & $2.2 \times 1.7^{c}$ & $-15^{c}$ & 0.67 & 5 & 870 \\
$\mathrm{SiO}(2-1)$ & IRAM-30 m $^{c}$ & 86.847 & 6 & 19.3 & 28 & - & 0.54 & 77 & 16 \\
$\mathrm{SiO}(3-2)$ & IRAM-30 m & 130.269 & 13 & 28.9 & 19 & - & 0.36 & 90 & 18 \\
$\mathrm{SiO}(5-4)$ & IRAM-30 m & 217.105 & 31 & 48.1 & 11 & - & 0.43 & 173 & 37 \\
$\mathrm{SiO}(5-4)$ & SMA & 217.105 & 31 & 48.1 & $1.7 \times 1.4$ & 67 & 0.67 & 36 & 415 \\
$\mathrm{SiO}(6-5)$ & IRAM-30 m & 260.518 & 44 & 57.7 & 9 & - & 0.36 & 189 & 42 \\
\hline
\end{tabular}

Notes. ${ }^{(a)}$ Frequencies and spectroscopic parameters of the molecular transitions have been extracted from the Jet Propulsion Laboratory molecular database (Pickett et al. 1998). ${ }^{(b)}$ For the molecular line observations the $1 \sigma$ noise is given per channel. ${ }^{(c)}$ The SiO $(1-0)$ emission has been successfully observed only with the VLA-D configuration.

This doubt calls for high-angular resolution observations of a reliable jet tracer. Silicon monoxide $(\mathrm{SiO})$ thermal emission is the best tool for this purpose: unlike other species such as $\mathrm{CO}$, it is associated with shocks inside jets, suffers minimal contamination from infalling envelopes or swept-up cavities, and traces regions close to protostars, which are heavily extincted even in the near- and mid-infrared regions (e.g. Codella et al. 2007; Lee et al. 2007). Only SiO line emission will allow us to probe the mass loss process and unambiguously determine the direction of the flows driven by the G24 cluster. The formation of $\mathrm{SiO}$ is attributed to the sputtering of $\mathrm{Si}$ atoms from charged grains in a magnetised C-shock with velocities higher than $20 \mathrm{~km} \mathrm{~s}^{-1}$ (Schilke et al. 1997; Gusdorf et al. 2008a,b). Although highangular resolution studies of $\mathrm{SiO}$ in high-mass star-forming regions still refer to a quite limited number of objects (Hunter et al. 1999; Cesaroni et al. 1999; Qiu et al. 2007; Zhang et al. 2007), they confirm the power of $\mathrm{SiO}$ in tracing the mass loss process in complex environments like those typical of the massive starforming regions.

In this paper, we present $\mathrm{SiO}(1-0)$ and $\mathrm{SiO}(5-4)$ images obtained with the NRAO ${ }^{1}$ Very Large Array (VLA) and SubMillimeter Array (SMA) ${ }^{2}$ as well as a complementary IRAM $^{3} 30-\mathrm{m}$ observations to unveil the mass loss process driven by the G24 cluster of high-mass YSOs.

\section{Observations}

\subsection{VLA}

The G24 cluster was observed with 27 antennas of the NRAO VLA to measure the $\mathrm{SiO}(1-0)$ line emission at $43.4 \mathrm{GHz}$ as well as the continuum emission. The observations were carried out in the $Q$-band with the D-configuration on August 10, 2008, and October 10, 2009, and with the C-configuration on August 14, 2009. The half power beam width (HPBW) of the antennas is $\sim 1^{\prime}$, which is the field-of-view of the images. The largest structure visible in the $\mathrm{C}+\mathrm{D}$-configuration is $\sim 43^{\prime \prime}$. The

\footnotetext{
1 The National Radio Astronomy Observatory is a facility of the National Science Foundation operated under cooperative agreement by Associated Universities, Inc.

2 The SubMillimeter Array is a joint project between the Smithsonian Astrophysical Observatory and the Academia Sinica Institute of Astronomy and Astrophysics, and is funded by the Smithsonian Institution and the Academia Sinica.

3 IRAM is supported by INSU/CNRS (France), MPG (Germany), and IGN (Spain).
}

phase reference centre of the observations was set to $\alpha_{2000}=$ $18^{\mathrm{h}} 36^{\mathrm{m}} 12^{\mathrm{s}} .660, \delta_{2000}=-07^{\circ} 12^{\prime} 10^{\prime} .^{\prime} 15$.

The $\mathrm{SiO}(1-0)$ line and the continuum were observed simultaneously with the correlator in 2 IF mode. The continuum emission was collected with a $200 \mathrm{MHz}$ bandwidth centred at $43.339 \mathrm{GHz}$. The $\mathrm{SiO}(1-0)$ line was covered by simultaneously using two slightly overlapping 6.25 $\mathrm{MHz}\left(43 \mathrm{~km} \mathrm{~s}^{-1}\right)$ bands $^{4}$ Unfortunately, technical problems did not allow us to properly trace the $\mathrm{SiO}(1-0)$ emission in the VLA-D configuration. The observations were performed in fast-switching mode. Bandpass and phase were calibrated by observing 1832-105, while the flux density scale was derived by observing $1331+305$ (3C 286). All data editing and calibration were carried out using the NRAO AIPS $^{5}$ package. The line cubes were obtained by subtracting the continuum from the line. Images were produced using natural weighting: Details of the synthesised cleaned beam, spectral resolution, and rms noise of the maps are given in Table 1 .

\subsection{SMA}

The target was observed in the $\mathrm{SiO}(5-4)$ line at $217.1 \mathrm{GHz}$ and in the continuum at $1.4 \mathrm{~mm}$ with the SMA using two different array configurations. Compact- and extended-array observations were taken on May 21 and July 19, 2008, respectively. The covered baselines have lengths between $\sim 8$ and $100 \mathrm{k} \lambda$ (compact) and 17 and $160 \mathrm{k} \lambda$ (extended). We used two spectral sidebands, both $2 \mathrm{GHz}$ wide, separated by $10 \mathrm{GHz}$, covering the frequency ranges of $215.4-217.4$ and $225.4-227.4 \mathrm{GHz}$, with a uniform spectral resolution of about $0.5 \mathrm{~km} \mathrm{~s}^{-1}$. The phase reference centre of the observations was set to $\alpha_{2000}=18^{\mathrm{h}} 36^{\mathrm{m}} 12^{\mathrm{s}} .565$, $\delta_{2000}=-07^{\circ} 12^{\prime} 10^{\prime} \cdot 90$.

Absolute flux calibration was derived from observations of Titan and Uranus. The bandpass of the receiver was calibrated by observations of the quasars 3C 279 and 3C 454.3. Amplitude and phase calibrations were achieved by monitoring 1743-038 and 1911-201. We estimated the flux-scale uncertainty to be better than $15 \%$. The visibilities were calibrated with the IDL superset MIR $^{6}$. Additional imaging and analysis was performed with MIRIAD (Sault et al. 1995) and GILDAS ${ }^{7}$. The continuum was constructed in the $(u, v)$-domain from the line-free channels both in the LSB and USB. Continuum maps were created by

\footnotetext{
4 The $12.5 \mathrm{MHz}$ bandwidth, needed to cover the whole $\mathrm{SiO}(1-0)$ profile, was not available during the observations.

5 http://www.aips.nrao.edu/index.shtml

6 http://cfa-www.harvard. edu/ cqi/mircook.html

7 http://wwW . iram. fr/IRAMFR/GILDAS
} 


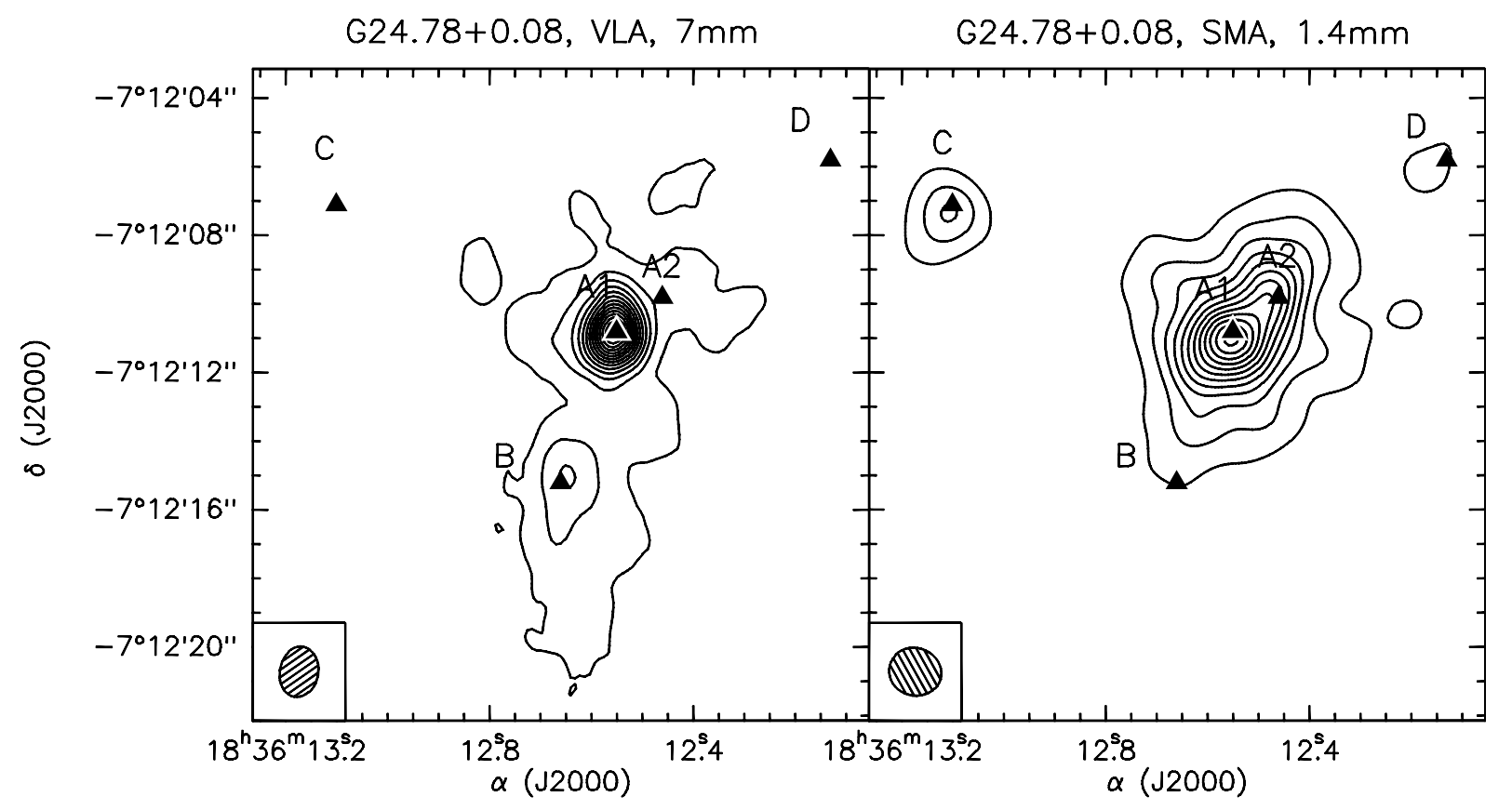

Fig. 1. Contour plots of the continuum emission at 7 (left panel) and $1.4 \mathrm{~mm}$ (right panel) imaged with the VLA and SMA interferometers, respectively. The black triangles mark the positions of the sources identified by Furuya et al. (2002) and Beltrán et al. (2004). The 1 $\sigma$ rms of the maps is 6.0 (at $1.4 \mathrm{~mm}$ ) and $0.8 \mathrm{mJy} \mathrm{beam}^{-1}$ (at $7 \mathrm{~mm}$ ), while first contours and steps correspond to $3 \sigma$ and $6 \sigma$. The ellipses in the bottom-left corners show the HPBW: 1". $5 \times 1^{\prime \prime} .4\left(\mathrm{PA}=74^{\circ}\right.$; SMA) and 1". $5 \times 1^{\prime \prime} .1\left(\mathrm{PA}=-11^{\circ}\right.$; VLA).

combining the data of both compact and extended configurations with the ROBUST parameter of Briggs (1995) set equal to zero, whereas $\mathrm{SiO}(5-4)$ line channel maps were created using natural weighting after continuum subtraction in the UV plane. Angular and spectral resolution and as map sensitivity are given in Table 1.

\subsection{IRAM 30-m}

Single-dish observations to prepare the interferometric observational campaign were obtained with the IRAM 30-m telescope at Pico Veleta (Granada, Spain). The observations were carried out on January 27, 2007 pointing the telescope towards the position used as phase centre for the SMA observations: $\alpha_{2000}=18^{\mathrm{h}} 36^{\mathrm{m}} 12^{\mathrm{s}} .565, \delta_{2000}=-07^{\circ} 12^{\prime} 10^{\prime} \cdot 90$. The pointing was checked by observing nearby planets or continuum sources and was found to be accurate to within $3-4$ ". The observations were made by position-switching in wobbler mode. As spectrometer, an autocorrelator split into different parts was used to allow simultaneous observations of four lines: $\mathrm{SiO}(2-1)$ at 86.8 , $(3-2)$ at $130.3,(5-4)$ at 217.1 , and $(6-5)$ at $260.5 \mathrm{GHz}$, respectively (see Fig. 3). The angular (HPBW) and the velocity resolutions provided by the backend, and the reached sensitivities are shown in Table 1 . The integration time $(\mathrm{ON}+\mathrm{OFF}$ source) was $70 \mathrm{~min}$, while the main beam efficiency varies from about 0.77 (at $87 \mathrm{GHz}$ ) to 0.48 (at $260 \mathrm{GHz}$ ). The spectra were calibrated with the standard chopper wheel method (uncertainty $\sim 10 \%$ ) and are reported here in units of main-beam temperature $\left(T_{\mathrm{MB}}\right)$.

\section{Results}

\subsection{Continuum emission}

Figure 1 shows the VLA and SMA maps of the continuum emission at 7 and $1.4 \mathrm{~mm}$, respectively, towards G24. Table 2 summarises the position and the peak intensity of the detected cores.
Table 2. Position and peak intensity of the continuum cores.

\begin{tabular}{|c|c|c|c|c|}
\hline Core & $\begin{array}{c}\alpha(\mathrm{J} 2000) \\
(\mathrm{h} \mathrm{m} \mathrm{s})\end{array}$ & $\begin{array}{c}\delta(\mathrm{J} 2000) \\
\left({ }^{\circ}, \prime \prime\right)\end{array}$ & $\begin{array}{l}I_{7 \mathrm{~mm}}^{\text {peak }} \\
(\mathrm{mJy}\end{array}$ & $\begin{array}{l}I_{1.4 \mathrm{~mm}}^{\text {peak }} \\
\left.\text { eam }^{-1}\right)\end{array}$ \\
\hline G24 A1 & 183612.55 & -071211.05 & 69 & 422 \\
\hline G24 B & 183612.65 & -071215.02 & 12 & - \\
\hline $\mathrm{G} 24 \mathrm{C}$ & 183613.11 & -071207.42 & - & 95 \\
\hline G24 D & 183612.17 & -071206.15 & - & 22 \\
\hline
\end{tabular}

Notes. The positions are based on the SMA image for all sources but B, which is detected only with the VLA.

Given the lower angular-resolution, the present continuum image at $1.4 \mathrm{~mm}$ does not add information with respect to our previous PdBI and SMA observations at similar wavelengths (1.3-1.4 mm; Beltrán et al. 2005, 2011), which are consistent with the new observations. On the other hand, the present VLA beam is slightly better than that of our previous observations at $7 \mathrm{~mm}\left(2^{\prime \prime} .3 \times 1^{\prime \prime} .7\right.$, Furuya et al. 2002), but the sensitivity is a factor 2 lower. Again, the present VLA results agree with the previously performed analysis. The source B has been detected at $7 \mathrm{~mm}$ and not at $1.4 \mathrm{~mm}$ is consistent with its evolved stage, which is associated with an ultra compact (UC) HII region (see e.g. Furuya et al. 2002). But the analysis of the continuum emission is beyond the scope of the present paper, and will not be further pursued.

\subsection{SiO outflows}

The outflow activity was previously detected using emission of CO isotopologues by Furuya et al. (2002) and Beltrán et al. (2011) with the PdBI and SMA interferometers. Two molecular outflows, both oriented in the northwest-southest direction, were imaged. One (hereafter called outflow A) associated with the region of the $\mathrm{A} 1+\mathrm{A} 2$ cores and is probably driven by $\mathrm{A} 2$. 


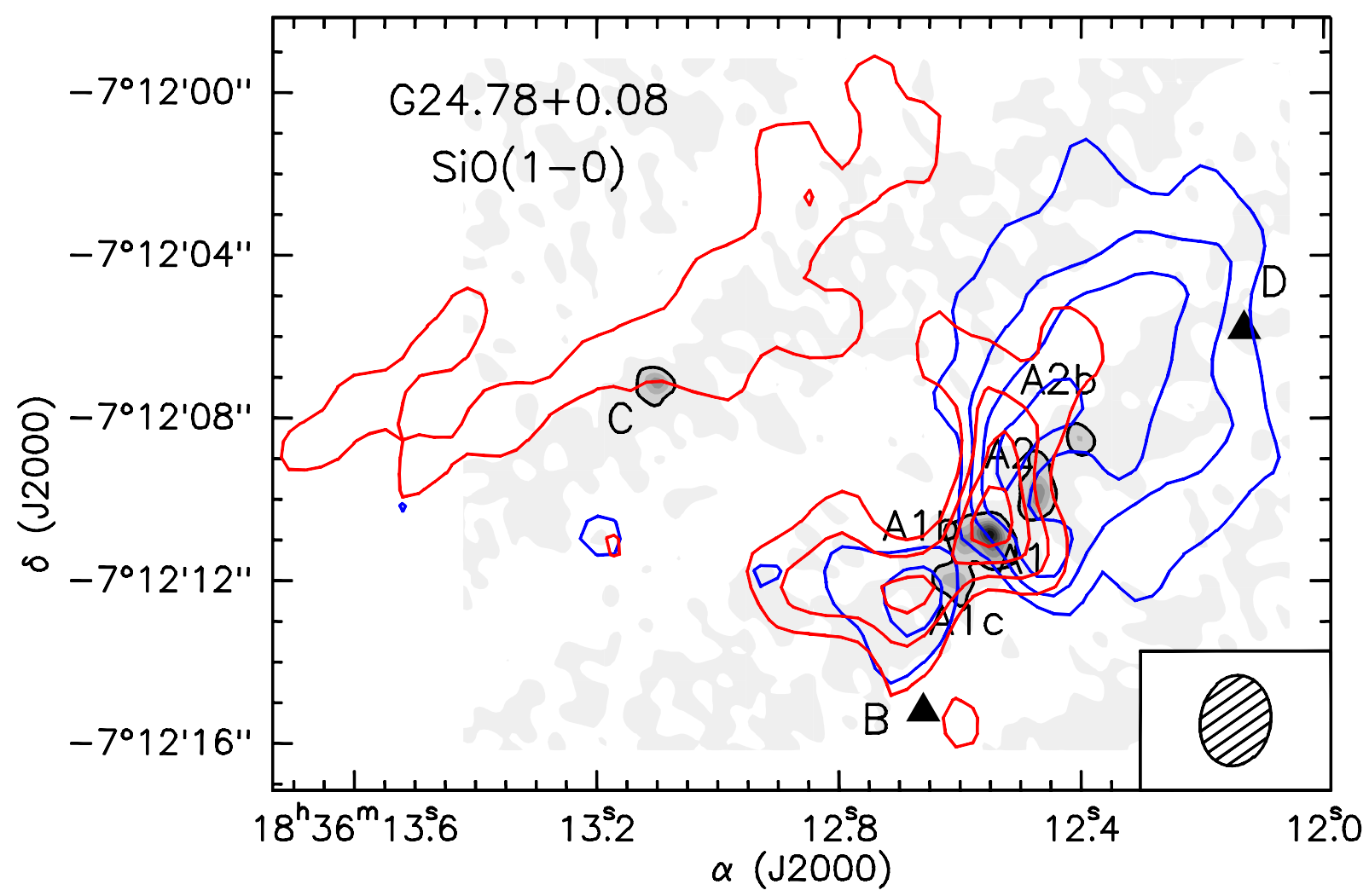

Fig. 2. Contour map of blue- and red-shifted $\mathrm{SiO}(1-0)$ VLA emission superimposed on the $1.3 \mathrm{~mm}$ continuum emission as observed at high angular resolution by Beltrán et al. (2011) using a very extended SMA configuration $\left(0{ }^{\prime} 55 \times 0\right.$ ' 44$)$. The sources of the G24.78+0.08 cluster are labelled following Furuya et al. (2002) and Beltrán et al. $(2004,2011)$. The $\mathrm{SiO}$ emission was averaged over the velocity intervals $(+80,+111) \mathrm{km} \mathrm{s}^{-1}$ and $(+111,+132) \mathrm{km} \mathrm{s}^{-1}$ for the blue- and red-shifted emission, respectively. The $1 \sigma \mathrm{rms}$ of the SiO maps is $1.2 \mathrm{mJy} \mathrm{beam}^{-1} \mathrm{~km} \mathrm{~s}^{-1}$. Contour levels range from $5 \sigma$ by steps of $3 \sigma$. The filled ellipse in the bottom-right corner shows the synthesised beam $(\mathrm{HPBW}): 2^{\prime \prime} .2 \times 1^{\prime \prime} .7\left(\mathrm{PA}=-15^{\circ}\right)$.

Another bipolar outflow is associated with core C. Figure 2 shows the maps of the integrated blue- and red-shifted $\mathrm{SiO}(1-0)$ emission observed with the VLA in the D configuration towards the G24 cluster, while Fig. 4 shows examples of the $\mathrm{SiO}(1-0)$ profiles observed towards the positions of A1 and of the blueand red-shifted $\mathrm{SiO}(1-0)$ emission peaks of the outflow A (see Table 3 ). The cloud systemic velocity is $+111 \mathrm{~km} \mathrm{~s}^{-1}$, according to Furuya et al. (2002). The $\mathrm{SiO}(1-0)$ lines are characterised by emission up to high velocities $\left(\sim 20-25 \mathrm{~km} \mathrm{~s}^{-1}\right.$ with respect to the cloud velocity) in the wings, i.e. velocities comparable with what is observed using $\mathrm{CO}$ isotopologues. For outflow A, the VLA data confirm a poorly collimated and extended blue-shifted NW lobe and a smaller and more collimated red-shifted SE lobe. As seen in CO (Beltrán et al. 2011), each lobe is characterised by the presence of a weaker extended counter-lobe. This could be a geometry effect because outflow A's main axis lies close to the plane of the sky. Alternatively, this could reflect the occurrence of a second outflow. For outflow $\mathrm{C}$, the $\mathrm{SiO}(1-0)$ map shows a very elongated red-shifted $\left(\sim 25 \mathrm{~km} \mathrm{~s}^{-1}\right.$ w.r.t. the systemic velocity) lobe: by dividing the length of the outflow by its width, we derive a collimation factor $f_{\mathrm{c}} \sim 4$. In this case, we detect no $\mathrm{SiO}$ blue-shifted emission, whereas the $\mathrm{CO}$ data of Beltrán et al. (2011) clearly show blue lobe, albeit smaller (by a factor 3 ) than the red lobe. This suggests that outflow $\mathrm{C}$ could be located close to the edge of the molecular cloud and therefore the blue-shifted gas could flow through a low-density region. This could also explain the lack of $\mathrm{SiO}$ emission, which is expected to trace the high-density collimated wind from the YSO.

Finally, no outflow activity has ever been observed towards source B, in agreement with its evolved stage, which is
Table 3. Positions of the SiO emission peaks in the VLA and SMA images.

\begin{tabular}{|c|c|c|}
\hline Peak & $\begin{array}{c}\alpha(\mathrm{J} 2000) \\
(\mathrm{h} \mathrm{m} \mathrm{s})\end{array}$ & $\begin{array}{c}\delta(\mathrm{J} 2000) \\
\left({ }^{\circ}{ }^{\prime}{ }^{\prime \prime}\right)\end{array}$ \\
\hline \multicolumn{3}{|c|}{$\mathrm{SiO}(1-0)-\mathrm{VLA}$} \\
\hline Red & 183612.55 & $-07 \quad 12 \quad 10.54$ \\
\hline Blue $^{a}$ & 183612.52 & $-07 \quad 1209.12$ \\
\hline \multicolumn{3}{|c|}{$\mathrm{SiO}(5-4)-\mathrm{SMA}$} \\
\hline Red & 183612.56 & $-07 \quad 12 \quad 10.50$ \\
\hline Blue & 183612.45 & -071210.30 \\
\hline
\end{tabular}

Notes. ${ }^{(a)}$ The $\mathrm{SiO}(1-0)$ blue-shifted emission is affected by absorption at low-velocities (see text).

associated with an UCHII region (Codella et al. 1997; Furuya et al. 2002). No $\mathrm{SiO}$ emission (or CO emission) has been detected towards core $\mathrm{D}$, which remains the most enigmatic object, bevause it is traced only by $\sim 1-3 \mathrm{~mm}$ continuum emission and is not detected in any molecule (Furuya et al. 2002; Beltrán et al. 2011). Our non-detection seems to confirm that D is a noncentrally-peaked core without active star formation.

The VLA image of the low-excitation $\left(E_{\mathrm{u}}=2 \mathrm{~K}\right) \mathrm{SiO}(1-0)$ emission is affected by absorption features ${ }^{8}$, which prevents us from drawing a definite picture of the morphology of the $\mathrm{SiO}$ outflow and from identifying the driving source. Figure 5 shows

\footnotetext{
8 Unfortunately, we lack the information on the $\mathrm{SiO}$ velocity between +111 and $+113 \mathrm{~km} \mathrm{~s}^{-1}$ towards the position of A1 because of technical problems.
} 


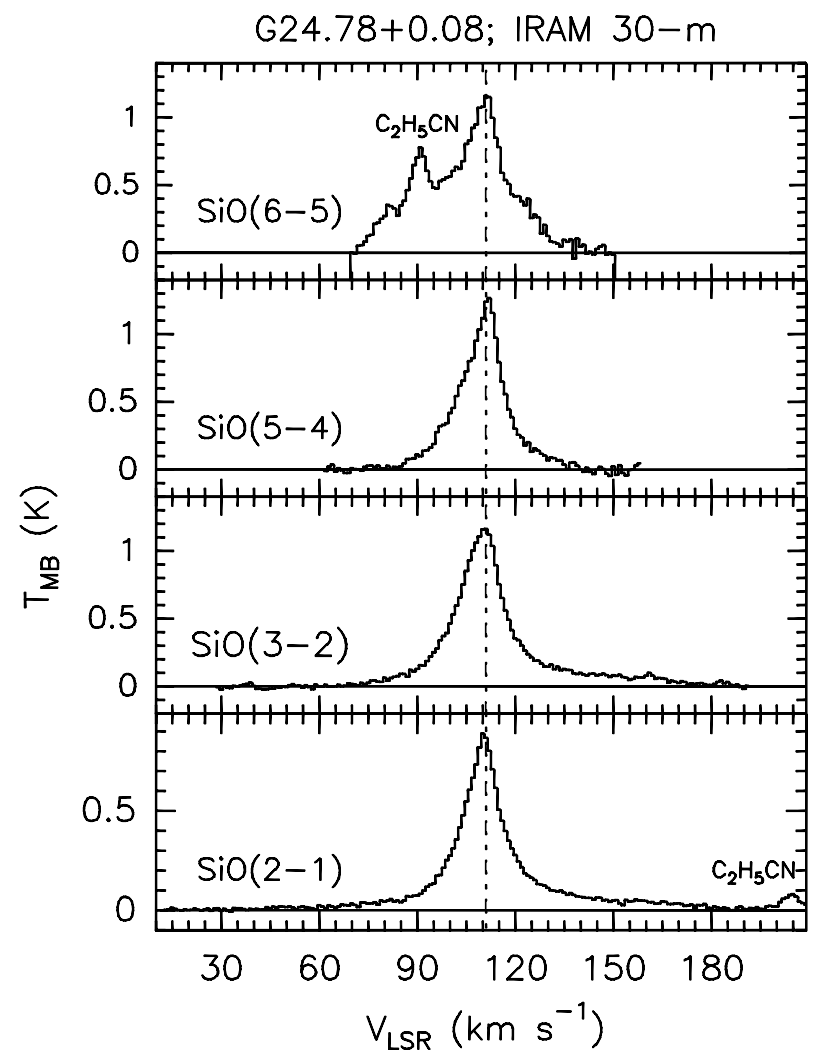

Fig. 3. $\mathrm{SiO}$ line profiles (in main-beam temperature, $T_{\mathrm{MB}}$, scale) observed with the IRAM 30-m antenna towards G24.78+0.08. The dashed lines stand for the systemic velocity $\left(+111.0 \mathrm{~km} \mathrm{~s}^{-1}\right)$. Two ethyl cyanide $\left(\mathrm{C}_{2} \mathrm{H}_{5} \mathrm{CN}\right)$ emission lines have been serendipitously detected at $86819.85 \mathrm{MHz}\left(10_{1,10}-9_{1,9} ; E_{\mathrm{u}}=24 \mathrm{~K}\right.$; bottom panel), and at $260535.69 \mathrm{MHz}\left(29_{5,25}-28_{5,24} ; E_{\mathrm{u}}=215 \mathrm{~K}\right.$; upper panel, blended with the $\mathrm{SiO}(6-5)$ blue wing).

a zoom-in of the central region of $\mathrm{G} 24.78+0.08$, centred on the cluster of the A1-A2 continuum sources. The green contours correspond to the narrow absorption blue-shifted feature (from +106 to $+110 \mathrm{~km} \mathrm{~s}^{-1}$, see Fig. 4) observed towards A1, against the hypercompact HII region imaged by Beltrán et al. (2007). This is ionised by a zero-age main-sequence star of spectral type O9.5 and mass of about $20 M_{\odot}$. The hypercompact HII region is very bright and therefore it is expected to observe colder molecular gas in absorption against it. Indeed, at cm-wavelengths, where the brightness temperature of $\mathrm{A} 1$ is $\sim 5 \times 10^{3} \mathrm{~K}$ in a $\sim 0$ ' 8 beam (Beltrán et al. 2007), the $\mathrm{NH}_{3}(2,2)$ spectral pattern has allowed us to trace the blue-shifted outflow lobe and the red-shifted gas infalling towards A1 in absorption (Beltrán et al. 2006). In conclusion, the VLA SiO(1-0) map calls for the spectral analysis of a higher-J $\mathrm{SiO}$ at higher frequencies, than those observed at SMA, where the brightness of the A1 hypercompact HII can be neglected.

Figure 6 shows the SMA maps of the integrated blue- and red-shifted $\mathrm{SiO}(5-4)$ emission. Only a hint of the outflow $\mathrm{C}$ is seen, and, as in the case of $\mathrm{SiO}(1-0)$, no emission has been observed towards cores B and D. On the other hand, the bipolar outflow A is clearly imaged, with the emission peaks lying closer to the A1+A2 system. The $\mathrm{SiO}(5-4)$ structure is consistent with that traced by the $\mathrm{SiO}(1-0)$ line. Table 3 reports the positions of the $\mathrm{SiO}$ emission peaks detected in the VLA and SMA images. The position of the red-shifted peak is basically the same (within the uncertainties) for the for the $\mathrm{SiO}(1-0)$ and (5-4) lines, whereas, given the absorption observed at low velocities that affects the $\mathrm{SiO}(1-0)$ map (see Fig. 4), the positions of the blue-shifted peaks in the two lines differ by $\sim 1^{\prime \prime}$. 6 . Example of $\mathrm{SiO}(5-4)$ spectra are shown in Fig. 4.

Figure 7 shows the maps of the $\mathrm{SiO}(5-4) / \mathrm{SiO}(1-0)$ intensity ratio, obtained after smoothing the maps to the same angular resolution, and derived where both emissions have a signal-to-noise $(S / N) \geq 3$. The velocities where $\mathrm{SiO}(1-0)$ shows absorption features (see Fig. 4) have not been taken into account. The red-shifted emission does not show a clear trend, while the $\mathrm{SiO}(5-4) / \mathrm{SiO}(1-0)$ ratio for the blue-shifted emission peaks in correspondence of A2, suggesting an increase of the $\mathrm{SiO}$ excitation conditions. Finally, in Fig. 8 we compare the $\mathrm{SiO}(5-4)$ profile observed with the IRAM 30-m antenna, with that obtained by integrating the SMA image over the IRAM beam (11"; blue histogram). The spectral shapes well match and, given the fluxscale uncertainties of the SMA (15\%) and IRAM 30-m (10\%), the line intensities also agree well, indicating that the SMA array recovers at least the $90 \%$ of the emission detected with the single-dish.

\subsection{The origin of the outflow $A$}

Which is the driving source of the outflow A? The VLA and SMA SiO images have been overlaid on the $1.3 \mathrm{~mm}$ continuum map obtained at sub-arcsecond angular resolution $\left(00^{\prime} 55 \times 00^{\prime} 44\right)$ by Beltrán et al. (2011), which gives the best picture so far available of the YSO population in the G24.78+0.08 cluster. It is possible to see that cores A1 and A2 are resolved into three (called A1, A1b, A1c) and two (A2, A2b) cores, respectively. By analysing the CO emission, Beltrán et al. (2011) suggested that the outflow $\mathrm{A}$ is actually powered by core A2, which is massive $\left(22 M_{\odot}\right)$ and associated with a hot-core $(\sim 180 \mathrm{~K})$ detected in $\mathrm{CH}_{3} \mathrm{CN}$, a typical hot-core tracer. The $\mathrm{SiO}$ images suggest that A1 is not the driving source of the outflow motions detected so far. Given the presence of several YSOs in the region, we cannot exclude additional fainter outflows. However, the peaks of the $\mathrm{SiO}(5-4)$ emission suggest that the geometrical centre of outflow $\mathrm{A}$, and thus its driving source, is located in the southern portion of the A2 core, which indeed is clearly elongated and probably hosts multiple YSOs. Additonal evidence comes from the position-velocity (PV) diagram of the $\mathrm{SiO}(5-4)$ transition along the NW-SE axis (see Fig. 9), which seems to confirm that A1 cannot be the driving source. Interestingly, the average velocity of the $\mathrm{SiO}$ emission increases as a function of distance from the geometrical centre, suggesting gas acceleration. Figure $10 \mathrm{com}-$ pares the PV diagram of $\mathrm{SiO}(5-4)$ with that derived from the CO(2-1) SMA data by Beltrán et al. (2011). We can clearly see that the $\mathrm{CO}$ emission (i) is completely absorbed by foreground material at low velocities and is affected by filtered extended emission, confirming the need of a tracer such as $\mathrm{SiO}$ to properly image the molecular outflow; and (ii) shows additional components with respect to what was traced by $\mathrm{SiO}$. Indeed, bright $\mathrm{CO}$ emission is seen at $\left(-5^{\prime \prime},+115 \mathrm{~km} \mathrm{~s}^{-1}\right)$, not traced by $\mathrm{SiO}$, as well as weak emission at $\left(+2^{\prime \prime},+113 \mathrm{~km} \mathrm{~s}^{-1}\right)$. In other words, the $\mathrm{CO}$ emission cannot be simply interpreted as an intensity-scaled version of the $\mathrm{SiO}$ emission. These findings are not surprising given what already found for the jets driven by Sun-like protostars, where, thanks to the smaller spatial scales that can be investigated, it is possible to see that $\mathrm{CO}$ traces not only the jet but also the walls of the cavity opened by the jet itself (e.g. Lee et al. 2007, see their Fig. 5). In summary, the present SiO maps support a formation mechanism for massive stars similar to that of their low-mass counterparts, where jets, clearly traced by $\mathrm{SiO}$ emission, create outflows of swept-up ambient gas traced by CO. 

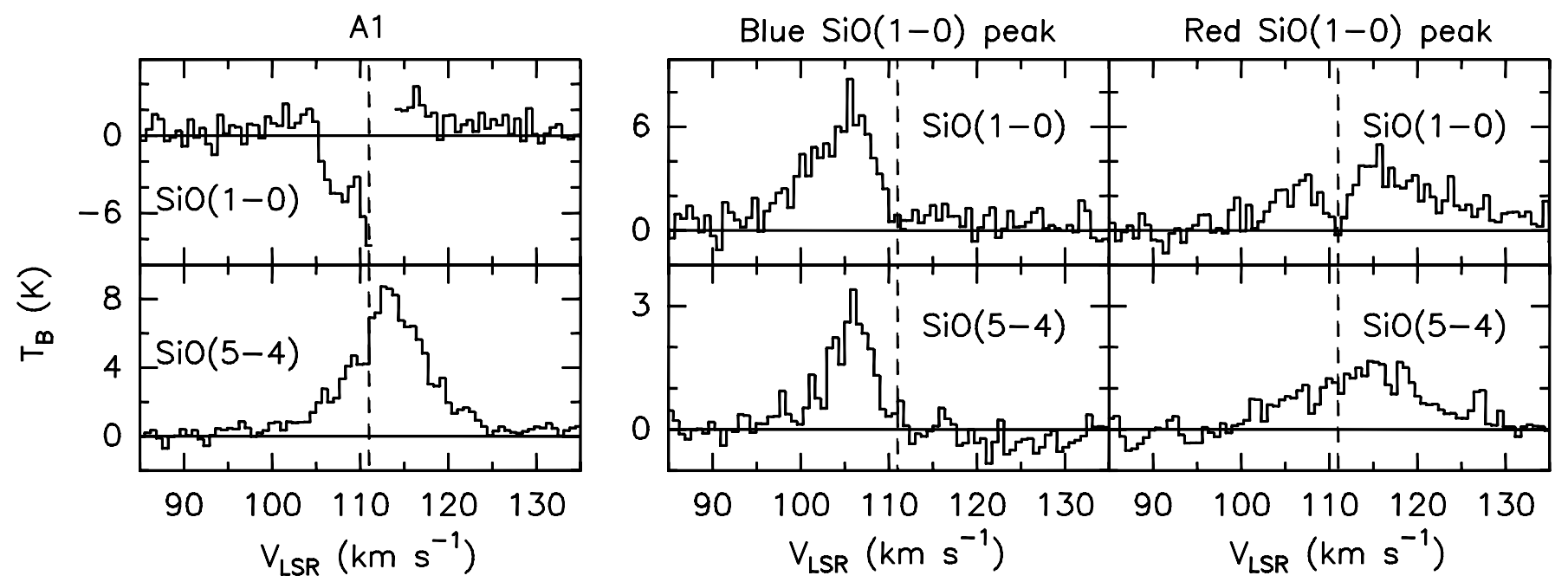

Fig. 4. Examples of $\mathrm{SiO}(1-0)$ and (5-4) line profiles (in brightness temperature $T_{\mathrm{B}}$, scale) observed towards A1 (left panel) and the peak positions of the blue- and red-shifted (middle and right panel) $\mathrm{SiO}(1-0)$ line emission. Dashed lines mark the systemic velocity $\left(+111 \mathrm{~km} \mathrm{~s}^{-1}\right)$.

G24.78+0.08, VLA

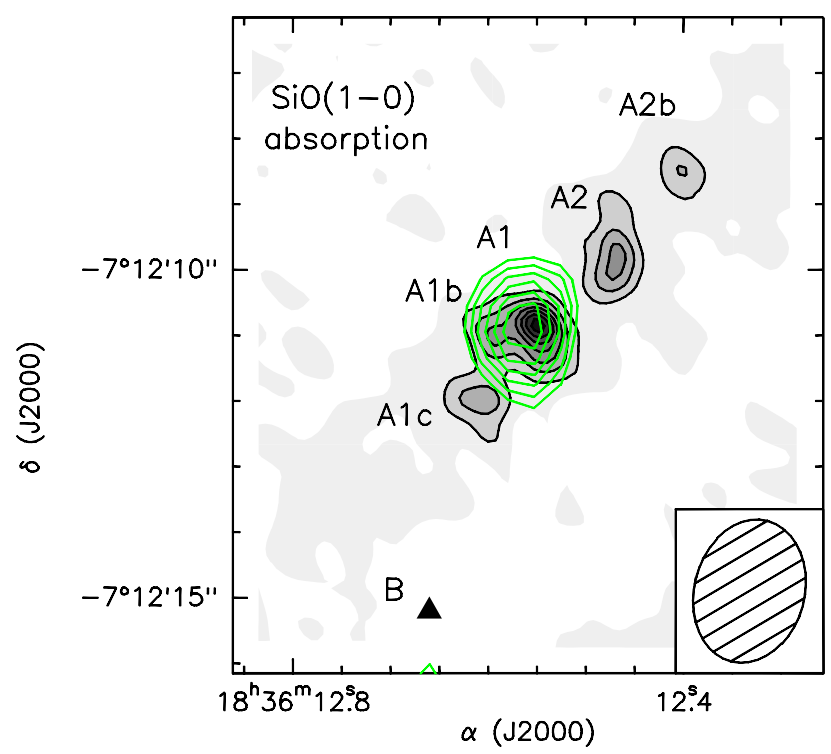

Fig. 5. Zoom-in of the central region of G24.78+0.08, where the cluster of A1-A2 continuum sources (grey scale) has been mapped at high angular resolution by Beltrán et al. (2011) using a very extended SMA configuration $\left(0{ }^{\prime} .55 \times 00^{\prime} 44\right)$. The spatial distribution of the blue-shifted (from +106 to $+110 \mathrm{~km} \mathrm{~s}^{-1}$ ) absorption (see Fig. 4) observed towards A1 is reported by the green contours. Contour levels range from $-10 \sigma$ to $-3 \sigma$ by steps of $1 \sigma\left(0.3 \mathrm{mJy}_{\text {beam }}^{-1} \mathrm{~km} \mathrm{~s}^{-1}\right)$. The filled ellipse in the bottom-right corner shows the synthesised beam (HPBW): 2 ". $2 \times 1$ 1.'7 $\left(\mathrm{PA}=-15^{\circ}\right)$.

The possibility of having an $\mathrm{SiO}$ jet is also supported by the extremely high velocity (up to $\sim 70 \mathrm{~km} \mathrm{~s}^{-1}$ with respect to the systemic velocity) of the $\mathrm{SiO}$ emission detected in the $J=2-1$ and 3-2 spectra thanks to the high sensitivities provided at these frequencies by the $30-\mathrm{m}$ antenna. Of course, in the present case, the angular resolution is not high enough to assess the occurrence of $\mathrm{SiO}$ jets. However, in analogy to the $\mathrm{SiO}$ jets in low-mass stars, one cannot rule out the possibility that the elongated $\mathrm{SiO}$ outflow does trace jet activity.
Table 4. List of the species and transitions serendipitously detected.

\begin{tabular}{lccc}
\hline \hline Transition & $\begin{array}{c}v^{a} \\
(\mathrm{GHz})\end{array}$ & $\begin{array}{c}E_{\mathrm{u}}{ }^{a} \\
(\mathrm{~K})\end{array}$ & $\begin{array}{c}S \mu^{2 a} \\
\left(\mathrm{D}^{2}\right)\end{array}$ \\
\hline $\mathrm{C}_{2} \mathrm{H}_{5} \mathrm{CN}\left(10_{1,10}-9_{1,9}\right)$ & 86.820 & 24 & 0.5 \\
$\left(\mathrm{CH}_{3}\right)_{2} \mathrm{CO}\left(18_{4,14}-17_{5,13}\right)-\mathrm{AE}$ & 215.881 & 111 & 655.7 \\
${ }^{13} \mathrm{CH}_{3} \mathrm{OH}-\mathrm{E}\left(4_{2,2}-3_{1,2}\right)$ & 215.887 & 45 & 3.5 \\
$\mathrm{CH}_{3} \mathrm{OCHO}-\mathrm{E}\left(20_{1,20}-19_{1,19}\right)$ & 215.892 & 298 & 53.0 \\
$\mathrm{CH}_{3} \mathrm{OCHO}-\mathrm{A}\left(19_{2,18}-18_{1,17}\right)$ & 216.360 & 109 & 7.2 \\
${ }^{13} \mathrm{CH}_{3} \mathrm{OH}-\mathrm{A}\left(10_{-2,9}-9_{-3,6}\right)$ & 216.370 & 162 & 2.7 \\
$\mathrm{H}_{2} \mathrm{~S}\left(2_{2,0}-2_{1,1}\right)$ & 216.710 & 84 & 2.0 \\
$\mathrm{C}_{2} \mathrm{H}_{3} \mathrm{CN}\left(23_{2,22}-22_{2,21}\right)$ & 216.937 & 134 & 996.4 \\
$\mathrm{CH}_{3} \mathrm{OH}-\mathrm{E}\left(5_{-1,4}-4_{-22}\right)$ & 216.946 & 56 & 1.1 \\
$\mathrm{CH}_{3} \mathrm{OCHO}-\mathrm{E}\left(17_{3,14}-16_{3,13}\right)$ & 216.959 & 286 & 43.7 \\
$\mathrm{CH}_{3} \mathrm{OCHO}-\mathrm{E}\left(20_{1,20}-19_{1,19}\right)$ & 216.965 & 111 & 52.8 \\
$\mathrm{CH}_{3} \mathrm{OCHO}-\mathrm{A}\left(20_{1,20}-19_{1,19}\right)$ & 216.966 & 111 & 52.8 \\
${ }^{13} \mathrm{CH}_{3} \mathrm{OH}-\mathrm{A}\left(10_{2,8}-9_{3,7}\right)$ & 217.400 & 162 & 2.7 \\
$\mathrm{HCC}^{13} \mathrm{CN}(24-23)$ & 217.420 & 130 & 346.4 \\
$\mathrm{C}_{2} \mathrm{H}_{5} \mathrm{CN}\left(29_{5,25}-28_{5,24}\right)$ & 260.536 & 215 & 417.0 \\
\hline
\end{tabular}

Notes. ${ }^{(a)}$ Frequencies and spectroscopic parameters of the molecular transitions have been extracted from the Jet Propulsion Laboratory molecular database (Pickett et al. 1998) for all transitions except those of methanol, which have been extracted from the Cologne Database for Molecular Spectroscopy (Müller et al. 2005).

\subsection{Other molecular species: the outflow and the hot core}

The 2-GHz-wide LSB bandwidth used to trace $\mathrm{SiO}(5-4)$ emission with the SMA allowed us to serendipitously observe several lines of different molecular species, which are listed in Table 4 and shown in Fig. 11. In particular, the LSB spectrum is dominated by broad $\mathrm{H}_{2} \mathrm{~S}\left(2_{2,0}-2_{1,1}\right)$ emission, which confirms that hydrogen sulphide is an excellent tracer of molecular outflows (Codella et al. 2003; Gibb et al. 2004). Figure 12 shows the integrated blue- and red-shifted $\mathrm{H}_{2} \mathrm{~S}$ emission from outflow A, while Fig. 9 shows the corresponding PV diagrams.

Outflow $\mathrm{A}$ is clearly seen in the $\mathrm{H}_{2} \mathrm{~S}$ image, which suggests (like $\mathrm{SiO}$ ) that the geometrical centre (and thus the driving source) is located in the southern portion of the A2 core. Interestingly, the $\mathrm{H}_{2} \mathrm{~S}$ outflow is definitely less collimated than the $\mathrm{SiO}(5-4)$ one, observed with the same angular resolution. $\mathrm{H}_{2} \mathrm{~S}$ and $\mathrm{SiO}$ show different behaviours also in the PV diagrams, 

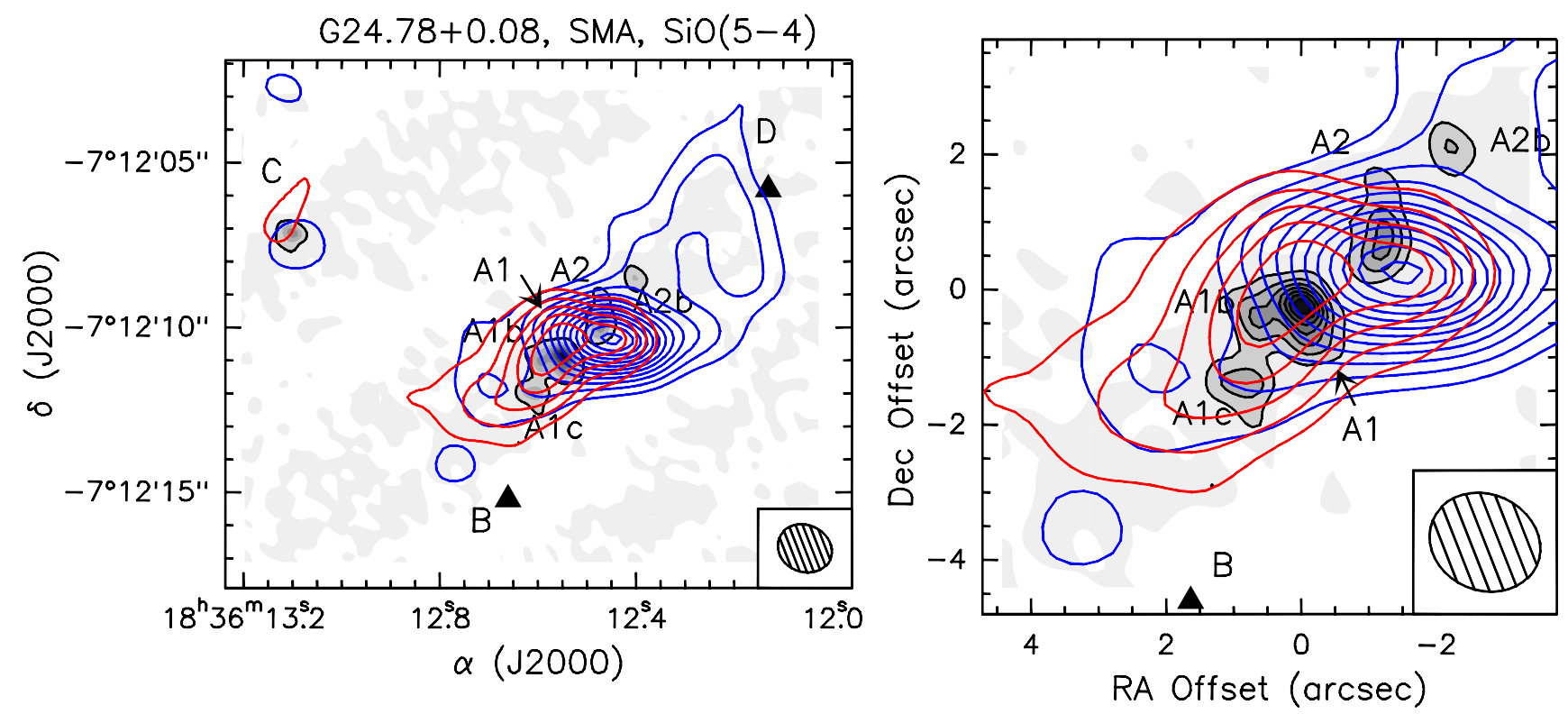

Fig. 6. Contour map of blue- and red-shifted $\mathrm{SiO}(5-4)$ SMA emission superimposed on the $1.3 \mathrm{~mm}$ continuum emission as observed at high angular resolution by Beltrán et al. (2011) using a very extended SMA configuration ( $0{ }^{\prime} 55 \times 0$. ' 44). The right panel reports a zoom-in of the central region. The sources of the G24.78+0.08 cluster are labeled following Furuya et al. (2002) and Beltrán et al. (2004, 2011). The SiO emission was averaged over the velocity intervals $(+89,+111) \mathrm{km} \mathrm{s}^{-1}$ and $(+111,+130) \mathrm{km} \mathrm{s}^{-1}$ for the blue- and red-shifted emission, respectively. The rms $1 \sigma$ of the $\mathrm{SiO}$ maps is $18 \mathrm{mJy}_{\text {beam }}^{-1} \mathrm{~km} \mathrm{~s}^{-1}$. Contour levels range from $5 \sigma$ by steps of $3 \sigma$. The filled ellipse in the bottom-right corner shows the synthesised beam $(\mathrm{HPBW}): 1^{\prime \prime} 7 \times 1^{\prime \prime} .4\left(\mathrm{PA}=67^{\circ}\right)$.

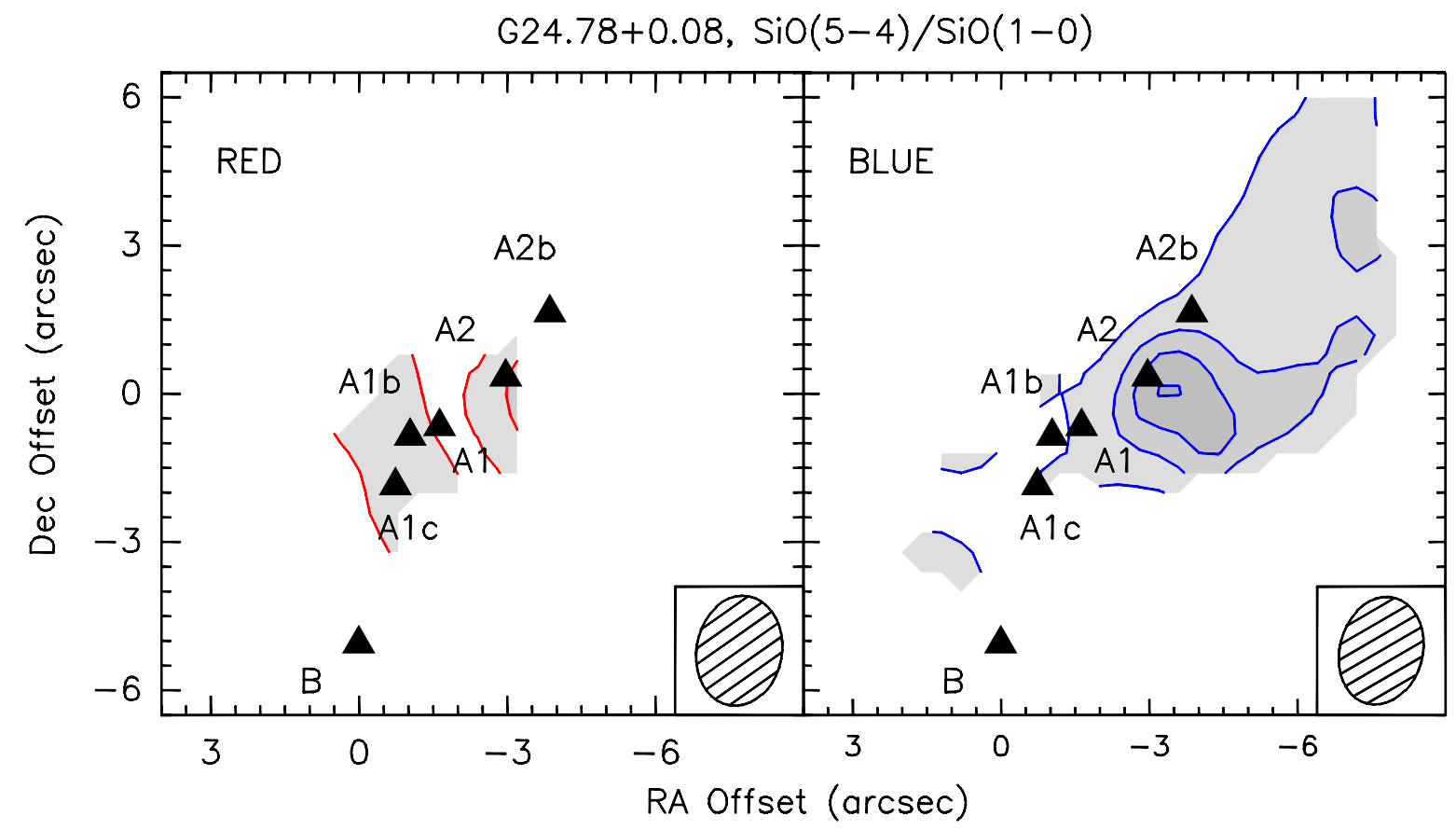

Fig. 7. $\mathrm{SiO}(5-4) / \mathrm{SiO}(1-0)$ intensity ratio (brightness temperature, $T_{\mathrm{B}}$, scale), derived where both emissions have an $S / N \geq 3$. For this comparison, the SMA image has been smoothed to the angular resolution of the VLA image. The velocities affected by $\mathrm{SiO}(1-0)$ absorption (see Fig. 4) have not been taken into account: right and left panels are for the blue $\left(+89,+105 \mathrm{~km} \mathrm{~s}^{-1}\right)$ and red $\left(+115,+130 \mathrm{~km} \mathrm{~s}^{-1}\right)$ emission, respectively. Contour levels range from 0.5 to 2.0 by steps of 0.5 . The sources of the G24.78+0.08 cluster are labelled following Furuya et al. (2002) and Beltrán et al. (2004, 2011).

where hydrogen sulphide does not show a clear increase of the average velocity with the distance from the driving source. These findings could be the signature of an additional fainter outflow driven by one of the A1+A2 YSOs that contributes to the observed emission. However, what we found agrees with an enhancement of the $\mathrm{H}_{2} \mathrm{~S}$ abundance as a consequence of the evaporation of the dust mantles in a shocked gas (e.g. van Dishoeck \& Blake 1998), whereas SiO comes from a definitely smaller region that is directly associated with the primary jet, where the refractory dust cores are disrupted as well.

In addition to $\mathrm{SiO}$ and $\mathrm{H}_{2} \mathrm{~S}$, we detected several highexcitation $\left(E_{\mathrm{u}}\right.$ between 45 and $\left.162 \mathrm{~K}\right)$ transitions of methanol isotopologues (see Table 4). Figure 11 shows the spectra observed towards the A2 position. The $\mathrm{CH}_{3} \mathrm{OH}$ line profiles are 


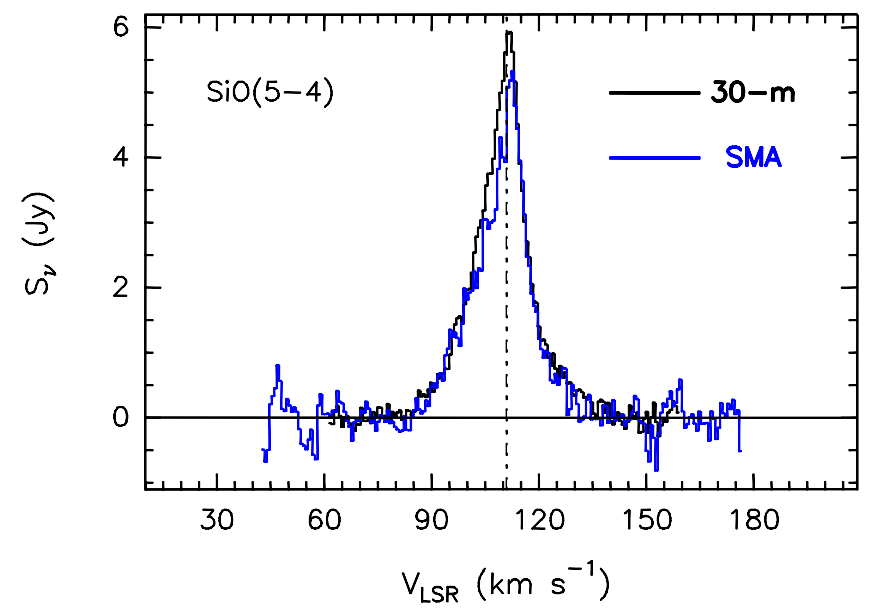

Fig. 8. Comparison between the $\mathrm{SiO}(5-4)$ line profile (in flux density, $S_{v}$, scale) observed with the IRAM 30-m antenna (black) and the spectrum obtained from the SMA data (blue) by integrating over a region equal to the IRAM 30-m beam (11"). For this comparison the IRAM $30-\mathrm{m}$ spectrum has been resampled to the spectral resolution of the SMA data $\left(0.67 \mathrm{~km} \mathrm{~s}^{-1}\right)$. The dashed lines stand for the systemic velocity $\left(+111.0 \mathrm{~km} \mathrm{~s}^{-1}\right)$.

narrower than those of $\mathrm{SiO}$ and $\mathrm{H}_{2} \mathrm{~S}$ and, although a weak component related to outflows cannot be excluded for ${ }^{12} \mathrm{CH}_{3} \mathrm{OH}$, it appears that methanol emission is dominated here by gas heated by YSOs. Indeed, from Fig. 9 one clearly sees that the PV diagram of methanol, outlining a compact circular pattern, is significantly different from that of $\mathrm{SiO}$. Most likely, the $\mathrm{CH}_{3} \mathrm{OH}$ molecules trace the hot core A2 (already imaged by Beltrán et al. 2005), and are released from dust mantles because of stellar irradiation. In particular, ${ }^{13} \mathrm{CH}_{3} \mathrm{OH}$ peaks at a position that matches the geometrical centre of the outflow well (see the solid vertical line in Fig. 9). Thus, the present observations suggest that a YSO lying in the SE of core A2 is heating the gas and driving the outflow A.

Finally, we also detected several lines in the SMA spectral window that are due to cyanoacetylene (in $\mathrm{HCC}^{13} \mathrm{CN}$ form) as well as to other complex molecular species usually considered as hot-core tracers: $\mathrm{CH}_{3} \mathrm{OCHO}$ (methyl formate), $\mathrm{C}_{2} \mathrm{H}_{3} \mathrm{CN}$ (vinyl cyanide), and, tentatively, one transition of $\left(\mathrm{CH}_{3}\right)_{2} \mathrm{CO}$ (acetone). Other complex species were reported by Beltrán et al. (2011, see their Table 1). Indeed, these emissions are characterised by high excitation ( $E_{\mathrm{u}}$ in the $109-298 \mathrm{~K}$ range) and compact spatial distribution (see the $\mathrm{C}_{2} \mathrm{H}_{3} \mathrm{CN}$ in Fig. 9 as an example), indicating an association with the hot core traced by ${ }^{13} \mathrm{CH}_{3} \mathrm{OH}$. Remarkable is the tentative detection of acetone, given that only recently this organic specie has been detected towards the hot molecular core Sagittarius B2(N-LMH) and in Orion BN/KL (Snyder et al. 2002; Friedel et al. 2005; Goddi et al. 2009).

\section{Physical conditions as traced by SiO}

\section{1. $\mathrm{SiO}(1-0)$ absorption}

From the $\mathrm{SiO}(1-0)$ absorption feature one can obtain an estimate of the excitation temperature of this transition. We assume that the absorption is due to the blue lobe of the outflow lying between the observer and the hypercompact HII region. Since the lobe is resolved in our maps, while the HII region is much smaller than the synthesised beam of the SiO images, the $\mathrm{SiO}(1-0)$ brightness temperature measured in the synthesised beam is a mixture of absorption (towards the HII region) and emission (from the rest of the beam):

$\Omega_{\mathrm{B}} T_{\mathrm{B}}=\left(\Omega_{\mathrm{B}}-\Omega_{\mathrm{S}}\right) T_{\mathrm{e}}+\Omega_{\mathrm{S}} T_{\mathrm{a}}$,

where $T_{\mathrm{e}}$ and $T_{\mathrm{a}}$ are the brightness temperatures of the $\mathrm{SiO}(1-0)$ line in emission and absorption, while $\Omega_{\mathrm{S}}$ and $\Omega_{\mathrm{B}}$ are the solid angle subtended by the HII region and the synthesised beam of the $\mathrm{SiO}(1-0)$ map. Moreover, $T_{\mathrm{e}}$ and $T_{\mathrm{a}}$ are given by

$T_{\mathrm{e}}=T_{\mathrm{ex}}\left(1-\mathrm{e}^{-\tau_{\mathrm{e}}}\right)$

$T_{\mathrm{a}}=\left(T_{\mathrm{ex}}-T_{\mathrm{HII}}\right)\left(1-\mathrm{e}^{-\tau_{\mathrm{a}}}\right)$,

with $T_{\text {ex }}$ excitation temperature of the $J=1-0$ transition and $T_{\mathrm{HII}}$ brightness temperature of the continuum emission from the hypercompact HII region. Here, we have assumed that $T_{\mathrm{ex}}$ is constant along the line of sight and across the region covered by the synthesised beam. After some algebra, one obtains the expression

$$
\begin{aligned}
T_{\mathrm{B}}= & T_{\mathrm{ex}}\left(1-\mathrm{e}^{-\tau_{\mathrm{e}}}\right)\left[1-\frac{\Omega_{\mathrm{S}}}{\Omega_{\mathrm{B}}} \frac{\mathrm{e}^{-\tau_{\mathrm{a}}}-\mathrm{e}^{-\tau_{\mathrm{e}}}}{1-\mathrm{e}^{-\tau_{\mathrm{e}}}}\right] \\
& -\frac{\Omega_{\mathrm{S}}}{\Omega_{\mathrm{B}}} T_{\mathrm{HII}}\left(1-\mathrm{e}^{-\tau_{\mathrm{a}}}\right) .
\end{aligned}
$$

We note that the $\Omega_{\mathrm{B}}$ is relatively small compared to the size of the outflow lobe, and hence the opacity should not depend significantly on the line of sight. Also, for obvious reasons, the HII region either lies behind the lobe or is enshrouded by it. Therefore, $\tau_{\mathrm{a}} \leq \tau_{\mathrm{e}}$ and $0 \leq \frac{\mathrm{e}^{-\tau_{\mathrm{a}}-\mathrm{e}^{-} \tau_{\mathrm{e}}}}{1-\mathrm{e}^{-\tau_{\mathrm{e}}}}<1$. Since $\frac{\Omega_{\mathrm{S}}}{\Omega_{\mathrm{B}}} \ll 1$, Eq. (4) can be written as

$T_{\mathrm{B}} \simeq T_{\mathrm{ex}}\left(1-\mathrm{e}^{-\tau_{\mathrm{e}}}\right)-\frac{\Omega_{\mathrm{S}}}{\Omega_{\mathrm{B}}} T_{\mathrm{HII}}\left(1-\mathrm{e}^{-\tau_{\mathrm{a}}}\right)$.

Most likely, the absorption and emission lines of sight are crossing similar amounts of gas, and we hence make the additional assumption that $\tau_{\mathrm{a}} \simeq \tau_{\mathrm{e}}$. By replacing Eq. (2) into Eq. (5), one can finally obtain an expression for $T_{\mathrm{ex}}$ :

$T_{\mathrm{ex}}=\frac{\Omega_{\mathrm{S}}}{\Omega_{\mathrm{B}}} T_{\mathrm{HII}} \frac{T_{\mathrm{e}}}{T_{\mathrm{e}}-T_{\mathrm{B}}}$

From our observations we measure absorption with $T_{\mathrm{B}} \simeq-6 \mathrm{~K}$ in a beam of $\Omega_{\mathrm{B}}=4.24 \operatorname{arcsec}^{2}$, while from the data of Beltrán et al. (2007) one obtains $\Omega_{\mathrm{S}} \simeq 0.0554 \operatorname{arcsec}^{2}$ and a sourceaveraged brightness temperature of the HII region at $7 \mathrm{~mm} T_{\mathrm{HII}} \simeq$ $960 \mathrm{~K}$. An estimate of $T_{\mathrm{e}}$ can be obtained from the $\mathrm{SiO}(1-0)$ spectra one beam away from the absorption peak: $T_{\mathrm{e}} \simeq 3-6 \mathrm{~K}$. With these numbers we obtain $T_{\mathrm{ex}} \simeq 4-8 \mathrm{~K}$. The brightness temperature of the continuum emission measured at $7 \mathrm{~mm}$ towards the HII region in our images is $\sim 12 \mathrm{~K}$, i.e. comparable to the value $\left(\frac{\Omega_{\mathrm{S}}}{\Omega_{\mathrm{B}}} T_{\mathrm{HII}} \simeq 12 \mathrm{~K}\right.$ ) estimated applying beam dilution to the high-resolution measurement of Beltrán et al. (2007): this proves that basically the entire continuum emission in our beam is due to free-free emission from the ionised gas, while dust emission is negligible.

\subsection{LVG analysis}

We ran the RADEX non-LTE model (van der Tak et al. 2007) with the rate coefficients for collisions with $\mathrm{H}_{2}$ reported by Turner et al. (1992) using the escape probability method for a plane-parallel geometry to fit the observed $\mathrm{SiO}$ line ratios and 


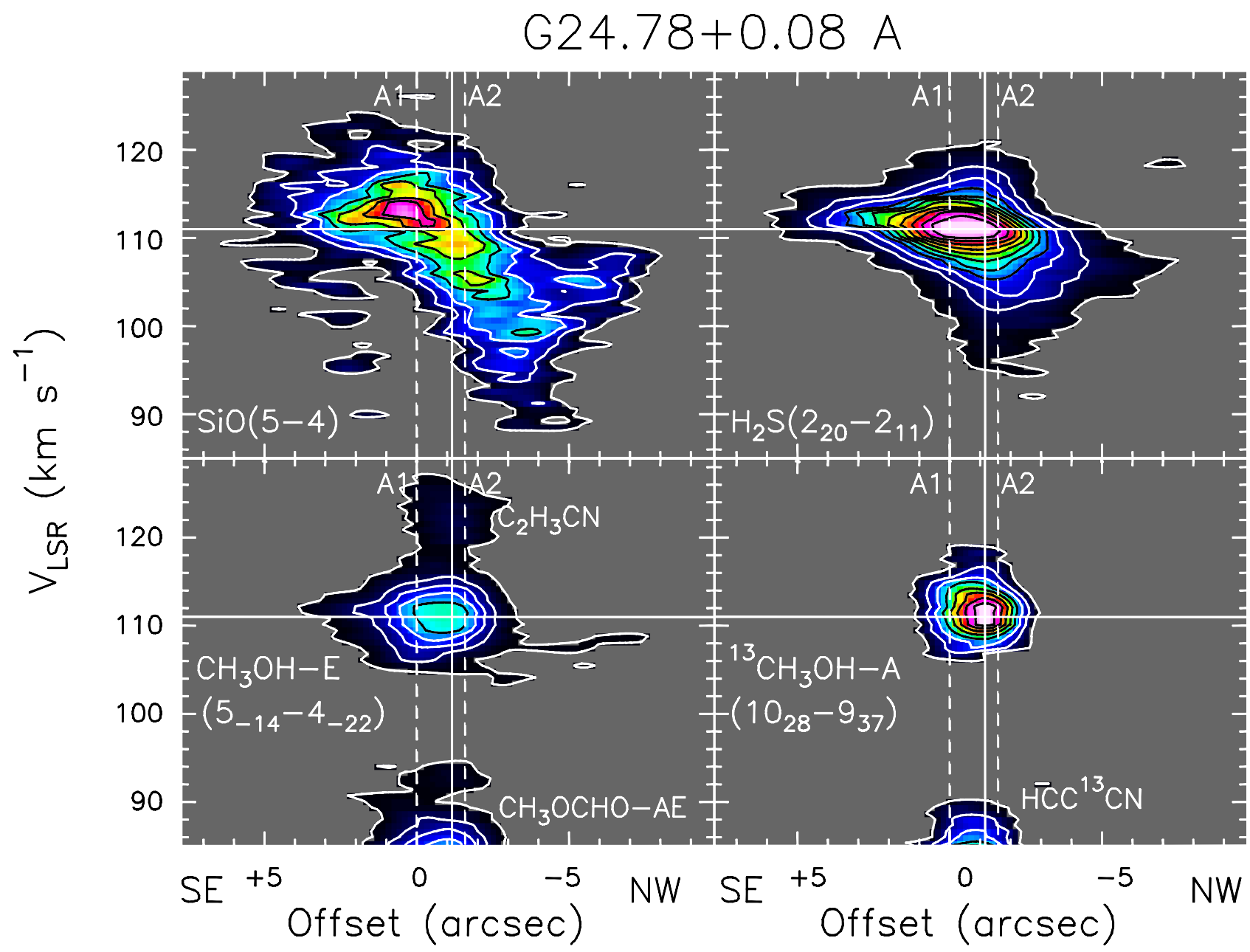

Fig. 9. Position-velocity cut of $\mathrm{SiO}(5-4), \mathrm{H}_{2} \mathrm{~S}\left(2_{2,0}-2_{1,1}\right), \mathrm{CH}_{3} \mathrm{OH}-\mathrm{E}\left(5_{-1,4}-4-22\right)$, and ${ }^{13} \mathrm{CH}_{3} \mathrm{OH}-\mathrm{A}\left(10_{2,8}-9_{3,7}\right)$ along the outflow $\mathrm{A}\left(\mathrm{PA}=-40^{\circ}\right)$. The position offsets are measured from the $\mathrm{A} 1$ position, positive towards northwest. The rms $1 \sigma$ is $20 \mathrm{mJy}^{-1}$ beam $^{-1}$. Contour levels range from $3 \sigma$ by steps of $3 \sigma$ for $\mathrm{SiO}$ and ${ }^{13} \mathrm{CH}_{3} \mathrm{OH}-\mathrm{A}$, and from $3 \sigma$ by steps of $10 \sigma$ for $\mathrm{H}_{2} \mathrm{~S}$ and $\mathrm{CH}_{3} \mathrm{OH}-\mathrm{E}$. Dashed vertical lines mark the position of the A1 and A2 YSOs, while the solid vertical line is for the average peak position of the typical hot-core tracers (see text). Solid horizontal line shows the systemic velocity $\left(+111.0 \mathrm{~km} \mathrm{~s}^{-1}\right)$.

brightness. We explored $\mathrm{H}_{2}$ densities from $10^{2}$ to $10^{8} \mathrm{~cm}^{-3}$, kinetic temperatures $T_{\text {kin }}$ from 50 to $500 \mathrm{~K}$, and an LVG optical depth parameter $n(\mathrm{SiO}) /(\mathrm{d} V / \mathrm{d} z)=N_{\mathrm{SiO}} / \Delta V$ ranging from $10^{12}$ to $10^{17} \mathrm{~cm}^{-2}\left(\mathrm{~km} \mathrm{~s}^{-1}\right)^{-1}$, i.e. from the fully optically thin to the optically thick regime. We used an FWHM linewidth of $10 \mathrm{~km} \mathrm{~s}^{-1}$, as suggested by the $\mathrm{SiO}$ spectra (see Fig. 4).

As a first step, we modelled the $\mathrm{SiO}$ emission observed with the IRAM 30-m telescope, using the $\mathrm{SiO}(2-1)$ and (5-4) transitions, which are sensitive to similar excitation conditions (see Table 1) as the lines observed with the interferometers (discussed below). The low- $J$ SiO lines, as already learned from previous studies of low-mass protostellar systems (e.g. Cabrit et al. 2007 and references therein), are not very sensitive to kinetic temperature. Figure 13 thus reports the solutions for the observed $\mathrm{SiO}(5-4) / \mathrm{SiO}(2-1)$ intensity ratio at the typical red$\left(+116 \mathrm{~km} \mathrm{~s}^{-1}\right)$ and blue-shifted $\left(+106 \mathrm{~km} \mathrm{~s}^{-1}\right)$ emission in the $n_{\mathrm{H}_{2}}-N(\mathrm{SiO})$ plane and for two extreme temperatures: 50 and $500 \mathrm{~K}$. The observed intensity ratio was corrected for beam dilution assuming an emitting source of 7 ", which is representative of the sizes of the outflows imaged with the VLA and SMA. To show how much the results depend on this assumption on emitting size, we also report the solutions for a smaller size (i.e. $3^{\prime \prime}$ ), which is the typical size of the brightest SiO clumps in G24. The dashed contours take into account the uncertainties associated with the intensity ratio. The $30-\mathrm{m}$ spectra do not provide any constraint on the kinetic temperature and column density, while densities of below $10^{5} \mathrm{~cm}^{-3}$ can be inferred.

On the other hand, we can obtain tight constraints on volume and column densities by analysing the $\mathrm{SiO}$ emission observed with the VLA and SMA arrays, after degrading the SMA map to the angular resolution of the VLA image. Figures 14 and 15 report the solutions for the observed $\mathrm{SiO}(1-0)$ and $\mathrm{SiO}(5-4)$ brightness temperatures at the same red- $\left(+116 \mathrm{~km} \mathrm{~s}^{-1}\right)$ and blue-shifted $\left(+106 \mathrm{~km} \mathrm{~s}^{-1}\right)$ velocities investigated using the 30-m spectra. We modelled the emission observed towards three positions: A1 (Fig. 14), as well as where the $J=1-0$ emission is only weakly affected by absorption, i.e. at the position of the red- and blue-shifted $\mathrm{SiO}(1-0)$ emission peaks (Fig. 15). As reported in Table 3, the positions of the red-shifted peak as traced by $\mathrm{SiO}(1-0)$ and (5-4), hence once considered the angular resolutions, agree well. We report the solutions found for the same kinetic temperatures used above to model the $30-\mathrm{m}$ emission $(50$ and $500 \mathrm{~K})$. We plot in black in Fig. 14 the contours corresponding to the the excitation temperature of the $\mathrm{SiO}(1-0)$ line estimated in Sect. 4.1 from the $\mathrm{SiO}(1-0)$ absorption feature observed towards A1.

In practice, the present LVG plots indicate for the three positions (i) volume densities between $10^{3}$ and $10^{5} \mathrm{~cm}^{-3}$; 


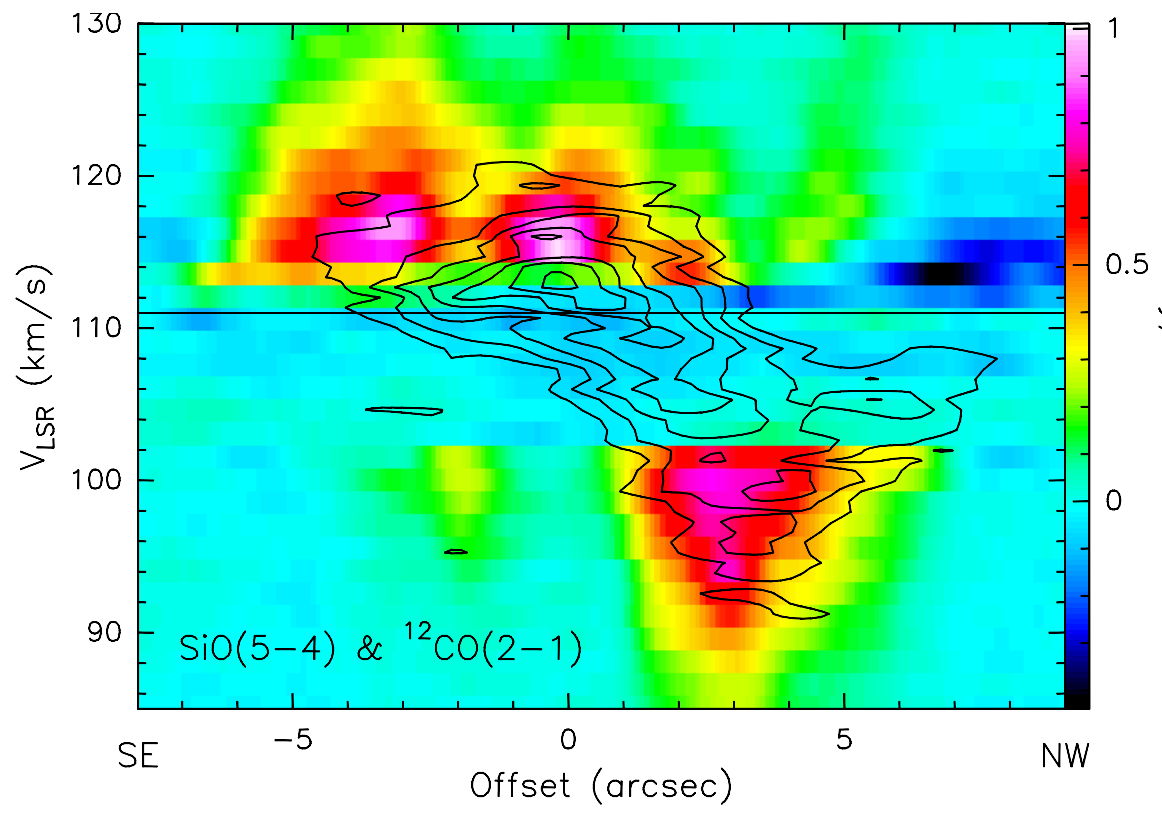

Fig. 10. Comparison between the positionvelocity cut of $\mathrm{SiO}(5-4)$, black contours, and that of $\mathrm{CO}(2-1)$ in colours, derived along the outflow $\mathrm{A}\left(\mathrm{PA}=-40^{\circ}\right)$ using the $\mathrm{CO}$ dataset presented by Beltrán et al. (2011). The solid horizontal line shows the systemic velocity $\left(+111.0 \mathrm{~km} \mathrm{~s}^{-1}\right)$.

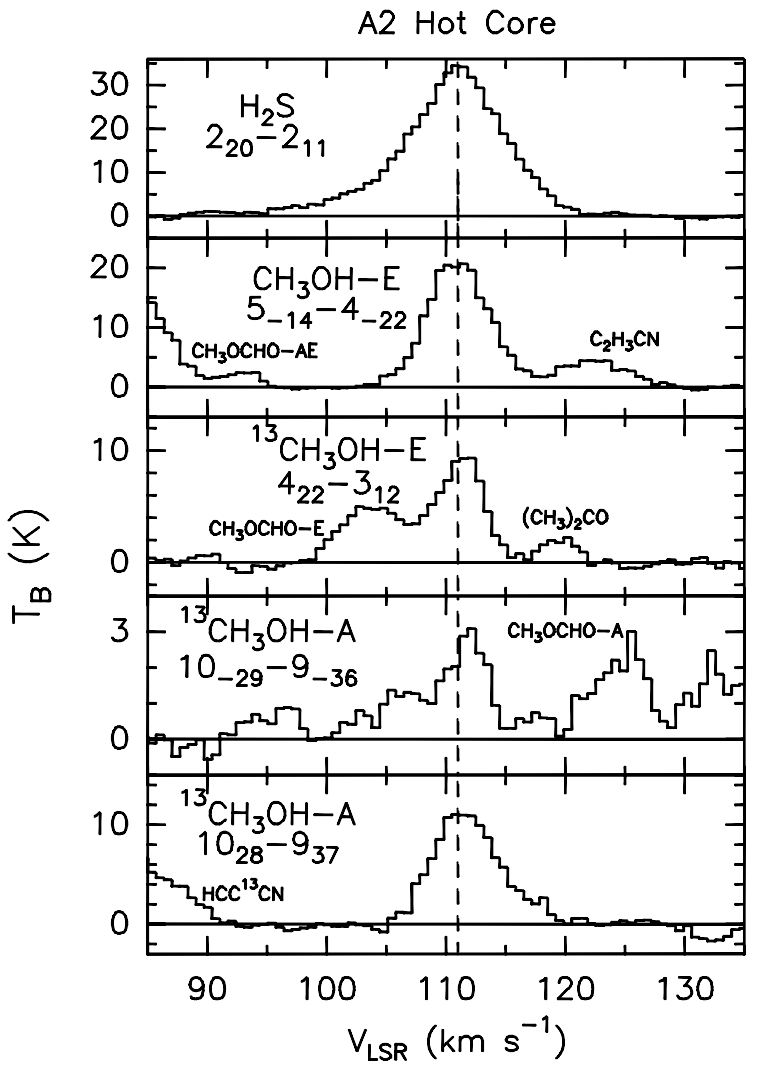

Fig. 11. $\mathrm{SiO}, \mathrm{H}_{2} \mathrm{~S}, \mathrm{CH}_{3} \mathrm{OH}$, and ${ }^{13} \mathrm{CH}_{3} \mathrm{OH}$ line profiles (in brightness temperature, $T_{\mathrm{B}}$, scale) observed towards the $\mathrm{A} 2$ hot core with the SMA. The dashed lines denote the systemic velocity $\left(+111 \mathrm{~km} \mathrm{~s}^{-1}\right)$. Several high-excitation lines due to typical tracers of hot-core chemistry (methyl formate, acetone, vinyl cyanide, cyanoacetilene) have also been detected (see Table 4).

and (ii) well-constrained $\mathrm{SiO}$ column density, in the $0.5-1 \times$ $10^{15} \mathrm{~cm}^{-2}$ range. These solutions are consistent with what was found using the 30-m IRAM SiO(5-4) and (2-1) spectra. Nisini et al. (2007) performed an LVG analysis of SiO emission towards the nearby prototypical Class 0 low-mass objects L1148 and L1157, using single-dish (IRAM, JCMT) data on angular

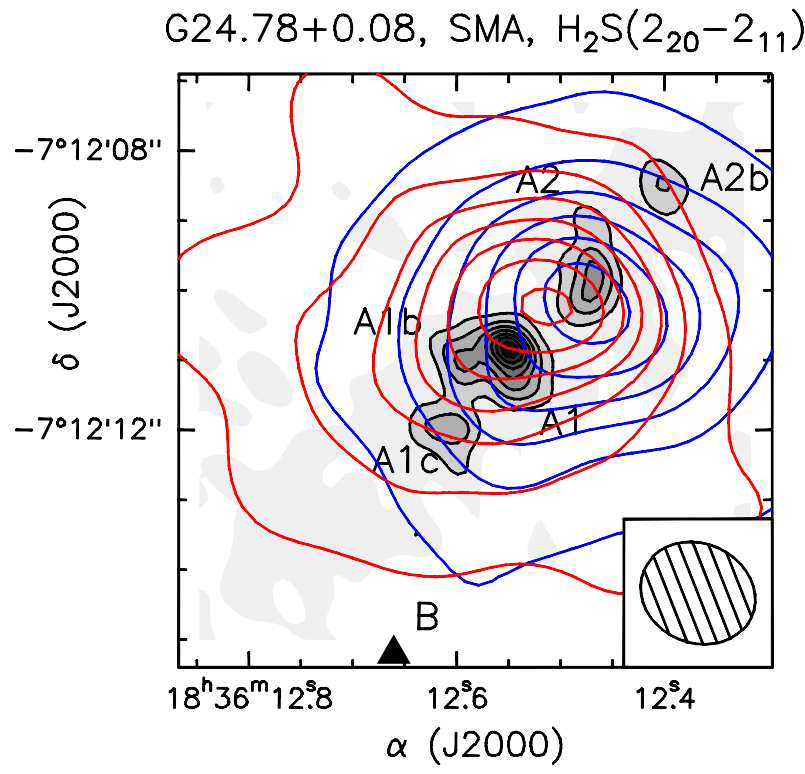

Fig. 12. Contour map of blue- and red-shifted $\mathrm{H}_{2} \mathrm{~S}\left(2_{2,0}-2_{1,1}\right)$ SMA emission superimposed on the $1.3 \mathrm{~mm}$ continuum emission as observed at high angular resolution by Beltrán et al. (2011) using a very extended SMA configuration $\left(0{ }^{\prime} 55 \times 0{ }^{\prime} 44\right)$. The sources of the G24.78+0.08 cluster are labelled following Furuya et al. (2002) and Beltrán et al. $(2004,2011)$. The $\mathrm{H}_{2} \mathrm{~S}$ emission was averaged over the velocity intervals $(+94,+111) \mathrm{km} \mathrm{s}^{-1}$ and $(+111,+124) \mathrm{km} \mathrm{s}^{-1}$ for the blue- and redshifted emission, respectively. The rms $1 \sigma$ is $18 \mathrm{mJy}_{\text {beam }}^{-1} \mathrm{~km} \mathrm{~s}^{-1}$. Contour levels range from $5 \sigma$ by steps of $10 \sigma$. The filled ellipse in the bottom-right corner shows the synthesised beam (HPBW): 1". $7 \times 1$ "' 4 $\left(\mathrm{PA}=67^{\circ}\right)$.

scales of $10^{\prime \prime}$. In that case as well, no tight constraints have been obtained for the kinetic temperature, whereas the volume densities were found to be between $10^{5}$ and $10^{6} \mathrm{~cm}^{-3}$. Moreover, Gusdorf et al. (2008a) modelled the $\mathrm{SiO}$ emission from L1157 using a C-shock code, which led to pre-shocked densities of $10^{4}-10^{5} \mathrm{~cm}^{-3}$. Thus, although completely different spatial scales are involved, the volume densities here inferred for G24 $\left(10^{3}-10^{5} \mathrm{~cm}^{-3}\right)$ appear to be consistent with those derived for L1448 and L1157. 

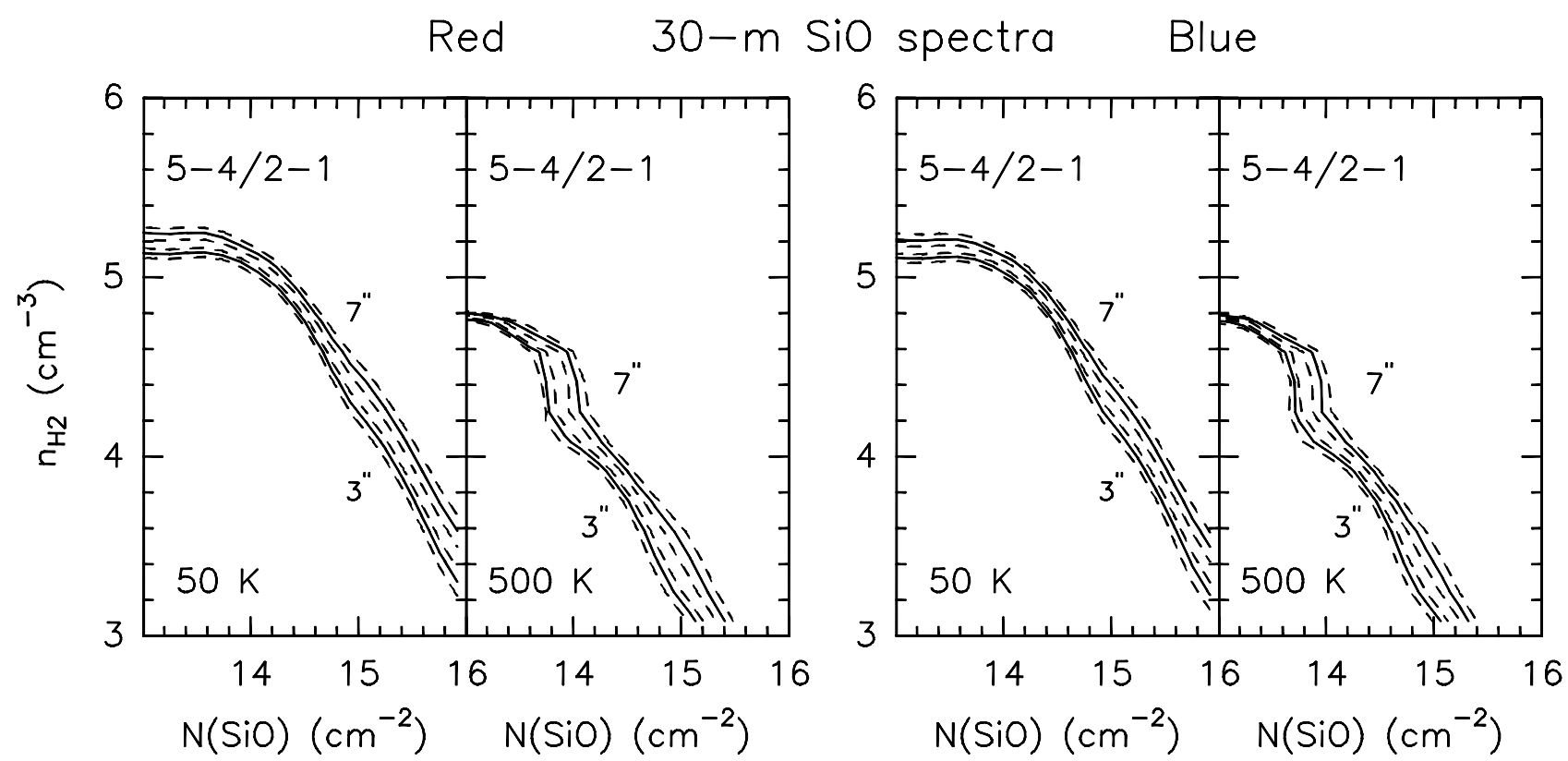

Fig. 13. Analysis of the $\mathrm{SiO}$ red-shifted line emission in the G24.78+0.08 outflow A observed with the IRAM 30-m antenna. The solutions for the observed $\mathrm{SiO}(5-4) / \mathrm{SiO}(2-1)$ intensity ratio are shown in the $n_{\mathrm{H}_{2}}-N(\mathrm{SiO})$ plot for non-LTE (RADEX) plane-parallel models at the labeled kinetic temperatures. Solid contours are for the measured ratios at the typical red- $\left(+116 \mathrm{~km} \mathrm{~s}^{-1}\right)$ and blue-shifted $\left(+106 \mathrm{~km} \mathrm{~s}^{-1}\right)$ velocities, after correction for a beam dilution derived assuming a source size of $3^{\prime \prime}$ and $7^{\prime \prime}$ (see text). The $\mathrm{SiO}(5-4) / \mathrm{SiO}(2-1)$ ratios are $0.21\left(3^{\prime \prime} ;+116 \mathrm{~km} \mathrm{~s}^{-1}\right)$, $0.19\left(3^{\prime \prime} ;+106 \mathrm{~km} \mathrm{~s}^{-1}\right), 0.27\left(7^{\prime \prime} ;+116 \mathrm{~km} \mathrm{~s}^{-1}\right)$, and $0.30\left(7^{\prime \prime} ;+106 \mathrm{~km} \mathrm{~s}^{-1}\right)$. Dashed contours take into account the uncertainties.
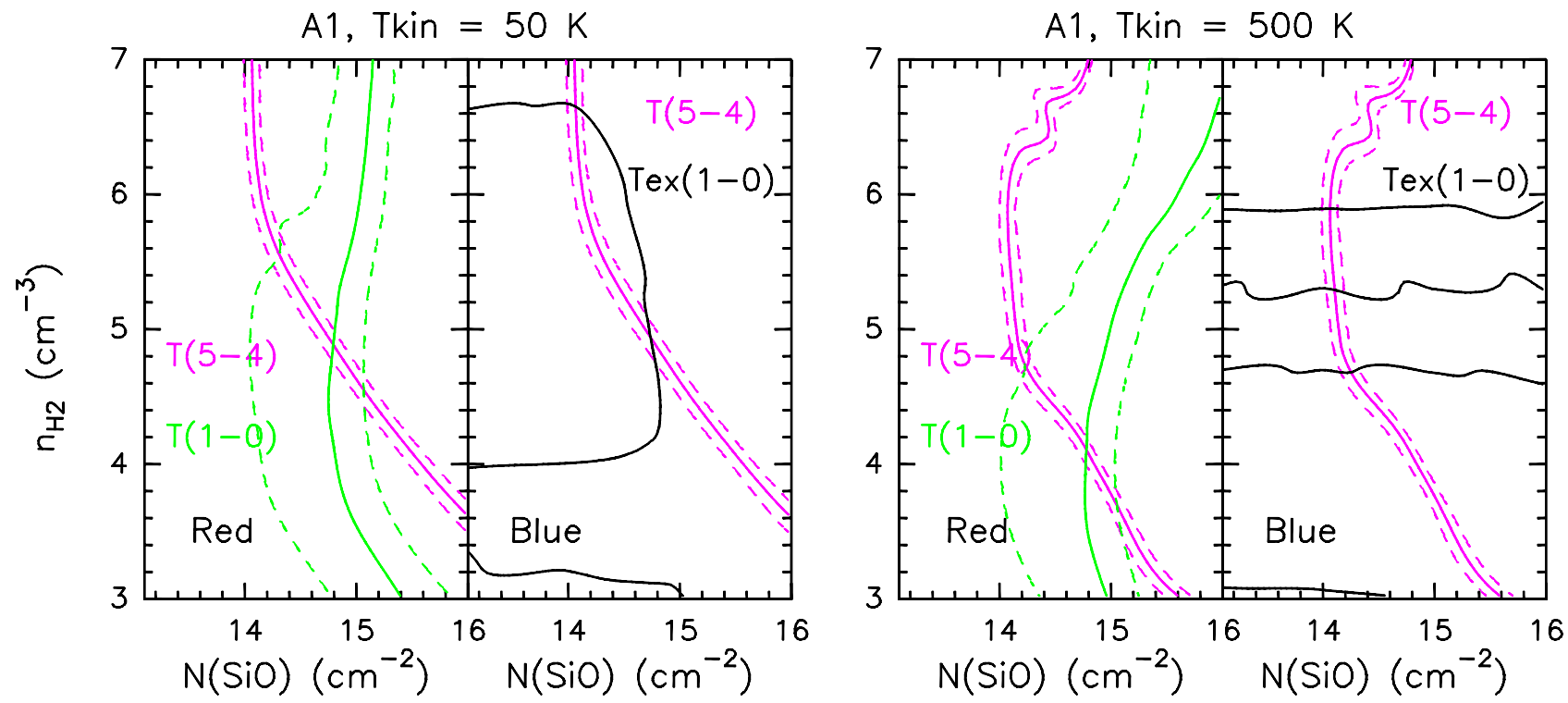

Fig. 14. Analysis of the $\mathrm{SiO}$ line blue- and red-shifted emissions towards A1 as observed with VLA and SMA (convolved to the angular resolution of the VLA image). The solutions for the observed $\mathrm{SiO}(1-0)$ and $\mathrm{SiO}(5-4)$ brightness temperatures (solid lines) at the typical red- $\left(+116 \mathrm{~km} \mathrm{~s}^{-1}\right.$ ) and blue-shifted $\left(+106 \mathrm{~km} \mathrm{~s}^{-1}\right)$ velocities are shown in the $n_{\mathrm{H}_{2}}-N(\mathrm{SiO})$ plane for non-LTE (RADEX) plane-parallel models at the labelled kinetic

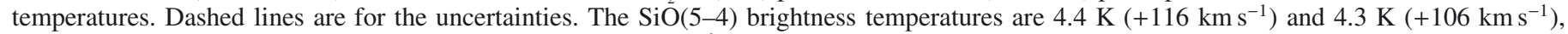
while the $\mathrm{SiO}(1-0)$ brightness temperature is $4.0 \mathrm{~K}\left(+116 \mathrm{~km} \mathrm{~s}^{-1}\right)$. Given the blue-shifted absorption observed towards A1, we plot in black the solutions corresponding to the derived $\mathrm{SiO}(1-0)$ excitation temperature $(\simeq 6 \mathrm{~K}$, see Sect. 4.1).

Assuming that the physical conditions reported above are representative of the whole $\mathrm{SiO}$ outflow (including where $\mathrm{SiO}(1-0)$ emission is affected by absorption), using a volume density of $10^{4} \mathrm{~cm}^{-3}$, the inferred size of the lobes $\left(7^{\prime \prime}\right)$, and the $\mathrm{G} 24$ distance $(7.7 \mathrm{kpc})$, the corresponding outflow mass is $\sim 40 M_{\odot}$. This implies, according to Beuther et al. (2002), Zhang et al. (2001, 2005), and López-Sepulcre et al. (2009, see their Fig. 5), a luminosity for the driving source of the $\mathrm{SiO}$ outflow of a few $10^{4} L_{\odot}$, corresponding to a late O-type
ZAMS YSO (e.g. Panagia 1973). If we conservatively assume $n_{\mathrm{H}_{2}}=10^{3} \mathrm{~cm}^{-3}$, the driving source is still a massive YSO, corresponding to a B2 spectral type. However, for consistency purposes, we cross-checked the outflow masses using the estimates of $\mathrm{SiO}$ column density and assuming a given $\mathrm{SiO}$ abundance relative to $\mathrm{H}_{2}$. The latter has a large uncertainty since $\mathrm{SiO}$ can be greatly enhanced in shocks. Assuming typical abundances of $10^{-8}-10^{-7}$ (i.e. $4-5$ orders of magnitude greater than that in dark clouds, $10^{-12}$, Ziurys et al. 1989), we inferred volume 

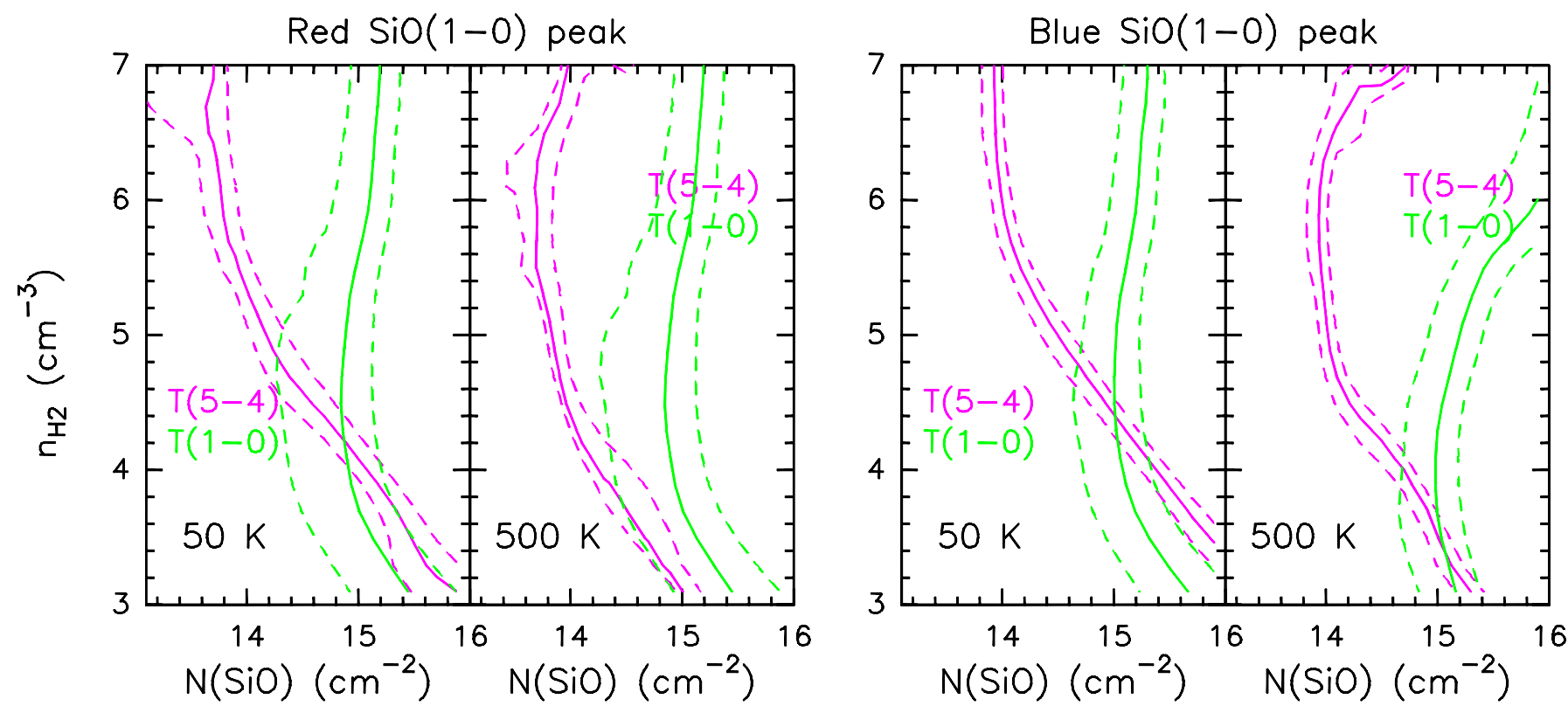

Fig. 15. Analysis of the $\mathrm{SiO}$ line blue- and red-shifted emissions towards the corresponding emission peaks of the $\mathrm{SiO}(1-0)$ map (see text), as observed with VLA and SMA (convolved to the angular resolution of the VLA image). The solutions for the observed $\mathrm{SiO}(1-0)$ and $\mathrm{SiO}(5-4)$ brightness temperatures at the typical red- $\left(+116 \mathrm{~km} \mathrm{~s}^{-1}\right)$ and blue-shifted $\left(+106 \mathrm{~km} \mathrm{~s}^{-1}\right)$ velocities are shown in the $n_{\mathrm{H}_{2}}-N(\mathrm{SiO})$ plane for nonLTE (RADEX) plane-parallel models at the labelled kinetic temperatures. The $\mathrm{SiO}(1-0)$ brightness temperatures are $4.7 \mathrm{~K}^{-1}\left(+116 \mathrm{~km} \mathrm{~s}^{-1}\right)$ and $6.1 \mathrm{~K}\left(+106 \mathrm{~km} \mathrm{~s}^{-1}\right)$, while the $\mathrm{SiO}(5-4)$ brightness temperatures are $1.1 \mathrm{~K}\left(+116 \mathrm{~km} \mathrm{~s}^{-1}\right)$ and $2.9 \mathrm{~K}\left(+106 \mathrm{~km} \mathrm{~s}^{-1}\right)$. Dashed lines are for the uncertainties.

densities between $10^{4}$ and $10^{5} \mathrm{~cm}^{-3}$, and thus again masses $\left(\sim 40-400 M_{\odot}\right)$ in agreement with an outflow driven by a late O-type ZAMS YSO.

\section{Conclusions}

We conducted a multiline $\mathrm{SiO}$ survey towards the $\mathrm{G} 24.78+0.08$ region, which is an excellent laboratory to study the process of high-mass star formation, because it is associated with YSOs in different evolutionary stages. After preliminary IRAM 30-m single-dish runs, we obtained high angular resolution images using the VLA and SMA interferometers. The main results can be summarised as follows:

1. High-velocity $\mathrm{SiO}$ emission (up to $25 \mathrm{~km} \mathrm{~s}^{-1}$ w.r.t. the systemic velocity, $+111 \mathrm{~km} \mathrm{~s}^{-1}$ ) reveals two collimated outflows driven by the $\mathrm{A} 2$ and $\mathrm{C}$ millimeter continuum massive cores. On the other hand, no $\mathrm{SiO}$ emission has been detected towards more evolved young stellar objects associated with an UCHII region (core B) or driven by the hypercompact (core A1) HII regions. Moreover core D shows no SiO emission, confirming its quiescent nature, without any signature of star formation.

2. The LVG analysis of the $\mathrm{SiO}$ emission reveals high-density gas $\left(10^{3}-10^{5} \mathrm{~cm}^{-3}\right)$, with clearly constrained $\mathrm{SiO}$ column densities $\left(\sim 10^{15} \mathrm{~cm}^{-2}\right)$. The average velocity of the $\mathrm{SiO}$ emission increases as a function of distance from the driving source, which suggests gas acceleration. Although the angular resolution is not high enough to demonstrate the occurrence of $\mathrm{SiO}$ jets, if we assume the standard approach that $\mathrm{SiO}$ is tracing shocks inside jets, it is reasonable to associate the observed collimated $\mathrm{SiO}$ structures with jet activity.

3. The driving source of the A2 outflow (i) has an estimated luminosity of $\geq 10^{4} L_{\odot}$, which is typical of a late O-type star; and (ii) is located at the centre of a hot molecular core (traced in the present data set by emission from methyl formate, vinyl cyanide, cyanoacetilene, and acetone) that rotates on a plane perpendicular to the outflow main axis.

4. To our knowledge, we obtained one of the first interferometric images of an $\mathrm{SiO}$ jet-like outflow from young $\geq 10^{4} L_{\odot}$ stars. High spatial resolution maps of $\mathrm{SiO}$ high-velocity emission driven from young $\geq 10^{4} L_{\odot}$ stars have have been so far obtained towards IRAS 18264-1152, 18566+0408, 20126+4104, and 23151+5912 (Cesaroni et al. 1999; Qiu et al. 2007; Zhang et al. 2007). IRAS 20126+4104 is probably the best example of a jet from a massive YSO so far observed; it is traced by $\mathrm{SiO}, \mathrm{H}_{2}$, and [FeII] emission (Caratti o Garatti et al. 2008). To conclude, the present SiO observations support the theory that O-type stars form according to a core accretion model, i.e. via a scaled-up picture typical of Sun-like star formation, where jets, well-traced by $\mathrm{SiO}$ emission, create outflows of accumulated and accelerated ambient gas that in turn is well traced by $\mathrm{CO}$.

Acknowledgements. We wish to thank the IRAM staff for the $30-\mathrm{m}$ observations performed in service mode. We are grateful to S. Cabrit for useful discussion and suggestions. We also thank the anonymous referee for comments and suggestions, which improved the work.

\section{References}

Bachiller, R., Peréz Gutiérrez, M., Kumar, M. S. N., \& Tafalla, M. 2001, A\&A, 372, 899

Beltrán, M. T., Cesaroni, R., Neri, R., et al. 2004, ApJ, 601, L187

Beltrán, M. T., Cesaroni, R., Neri, R., et al. 2005, A\&A, 435, 901

Beltrán, M. T., Cesaroni, R., Codella, C., et al. 2006, Nature, 443, 427

Beltrán, M. T., Cesaroni, R., Moscadelli, L., \& Codella, C. 2007, A\&A, 471, L13

Beltrán, M. T., Cesaroni, R., Zhang, Q., et al. 2011, A\&A, 532, A91

Beuther, H., Schilke, P., Sridharan, T. K., et al. 2002, A\&A, 383, 892

Bonnel, I. A., Larson, R. B., \& Zinnecker, H. 2007, Protostars \& Planets V, eds. B. Reipurth, D. Jewitt, \& K. Keil (University of Arizona Press), 149 Briggs, D. 1995, Ph.D. Thesis, New Mexico Inst. Mining \& Tech.

Caratti o Garatti, A., Froebrich, D., Eislöffel, J., Giannini, T., \& Nisini, B. 2008, A\&A, 485, 137 
Cabrit, S., Codella, C., Gueth, F., et al. 2007, A\&A, 468, L29

Cesaroni, R., Felli, M., Jenness, T., et al. 1999, A\&A, 345, 949

Cesaroni, R., Codella, C., Furuya, R. S., \& Testi, L. 2003, A\&A, 401, 227

Cesaroni, R., Neri, R., Olmi, L., et al. 2005, A\&A, 434, 1039

Codella, C., Testi, L., \& Cesaroni, R. 1997, A\&A, 325, 282

Codella, C., Bachiller, R., Benedettini, M., \& Caselli, P. 2003, MNRAS, 341, 707

Codella, C., Cabrit, S., Gueth, F., et al. 2007, A\&A 462, L53

Friedel, D. N., Snyder, L. E., Remijan, A. J., \& Turner, B. E. 2005, ApJ, 632, L95

Furuya, R. S., Cesaroni, R., Codella, C., et al. 2002, A\&A, 390, L1

Gibb, A. G., Wyrowski, F., \& Mundy, L. G. 2004, ApJ, 616, 301

Goddi, C., Greenhill, L. J., Humphreys, E. M. L., et al. 2009, ApJ, 691, 1254

Gusdorf, A., Cabrit, S., Flower, D. R., \& Pineau Des Forêts, G. 2008a, A\&A 482, 809

Gusdorf, A., Pineau Des Forêts, G., Cabrit, S., \& Flower, D. R. 2008b, A\&A 490, 695

Hunter, T. R., Testi, L., Zhang, Q., \& Sridharan, T. K. 1999, AJ, 118, 477

Lee, C.-F., Ho, P. T. P., Beuther, H., et al. 2007a, ApJ, 659, L499

Lee, C.-F., Ho, P. T. P., Palau, A., et al. 2007b, ApJ, 670, 1188

López-Sepulcre, A., Codella, C., Cesaroni, R., Marcelino, N., \& Walmsley, C. M. 2009, A\&A, 499, 811

McKee, C. F., \& Tan, J. C. 2002, Nature, 416, 59
McKee, C. F., \& Tan, J. C. 2003, ApJ, 585, 850

Moscadelli, L., Goddi, C., Cesaroni, R., Beltrán, M. T., \& Furuya, R. S. 2007, A\&A, 472, 867

Müller, H. S. P., Schöier, F. L., Stutzki, J., \& Winnewisser, G. 2005, J. Mol. Struct., 742, 215

Panagia, N. 1973, AJ, 78, 929

Pickett, H. M., Poynter, R. L., Cohen, E. A., et al. 1998, J. Quant. Spectrosc. \& Rad. Transfer, 60, 883

Qui, K., Zhang, Q., Beuther, H., \& Yang, J. 2007, ApJ, 654, 361

Sault, R. J., Teuben, P. J., \& Wright, M. C. H. 1995, in Astronomical Data Analysis Software and Systems IV, ASP Conf. Ser., 77, 433

Schilke, P., Walmsley, C. M., Pineau des Forêts, G., \& Flower, D. R. 1997, A\&A 321,293

Snyder, L. E., Lovas, F. J., Mehringer, D. M., et al. 2002, ApJ, 578, 245

Turner, B. E., Chan, K. W., Green, S., \& Lubowich, D. A. 1992, ApJ, 399, 114 van der Tak, F. F. S., Black, J. H., Schøier, F. L., et al. 2007, A\&A, 468, 627 van Dishoeck, E. F. \& Blake, G. A. 1998, ARA\&A, 36, 317

Vig, S., Cesaroni, R., Testi, L., Beltrán, M. T., \& Codella, C. 2008, A\&A, 488, 605

Zhang, Q., Hunter, T. R., Brand, J., et al. 2001, ApJ, 552, L167

Zhang, Q., Hunter, T. R., Brand, J., et al. 2005, ApJ, 625, 864

Zhang, Q., Sridharan, T. K., Hunter, T. R., et al. 2007, A\&A, 470, 269

Ziurys, L.-M., Friberg, P., \& Irvine, W. M. 1989, ApJ, 343, 201 